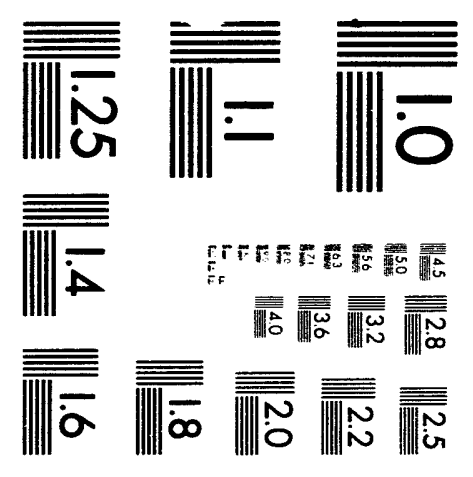



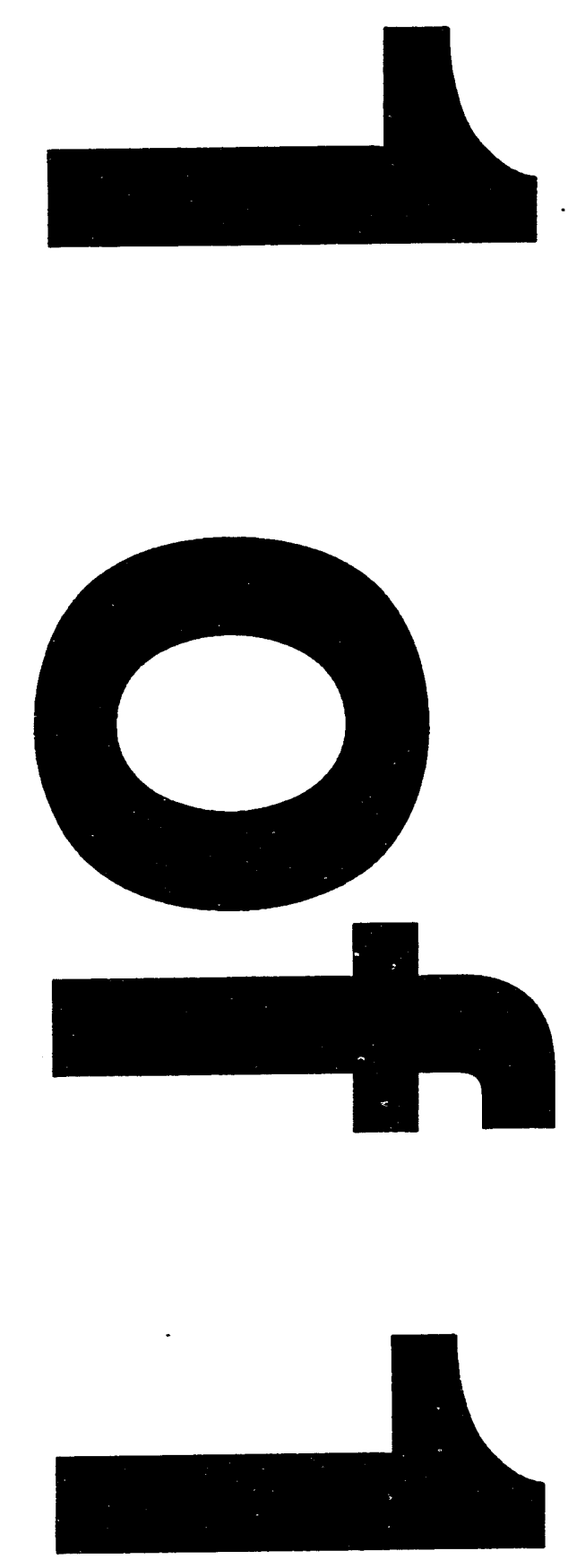
LBL-34837

CBP -45

\title{
A THEORY OF TWO-BEAM ACCELERATION OF CHARGED PARTICLES IN A PLASMA WAVEGUIDE* ${ }^{*}$
}

\author{
A.O. Ostrovsky \\ Kharkov Institute of Physics and Technology \\ Akademicheskayay St. 1 \\ Kharkov 310108 Ukraine
}

*Work performed for the Lawrence Berkeley Laboratory

tWork supported by the Director, Office of Energy Research, Office of High Energy and Nuclear Physics, Division of Hight Energy Physics, of the U.S. Department of Energy under Contract No. DE-AC03-76SF00098 


\section{Introduction}

The progress made in recent years in the field of high-current relativistic electron beam (REB) generation [1-3] has aroused a considerable interest in studying REB potentialities for charged particle acceleration with a high acceleration rate $T=100 \mathrm{MeV} / \mathrm{m}$. It was proposed, in particular, to employ high-current REB in two-beam acceleration schemes (TBA) [4-12]. In these schemes high current REB (driving beam) excites intense electromagnetic waves in the electrodynamic structure which, in their turn, accelerate particles of the other beam (driven beam).

The TBA schemes can be divided into two groups. The first group includes the schemes, where the two beams (driving and driven) propagate in different electrodynamic structures coupled with each other through the waveguides which ensure the microwave power transmission to accelerate driven beam particles $[4,6,11]$. Note that in these schemes a free-electron laser (FEL) or a relativistic klystron are considered as possible microwave power sources. These schemes differ from the traditional accelerator by the employment of only one intense beam over the whole length of the accelerating structure, whereas in traditional accelerators many beams are used to control different microwave radiation sources (klystrons).

The second group includes the TBA schemes, where the driving and driven beams propagate in a one electrodynamic structure $[5,7,10,12]$. In order to provide high chargedparticle acceleration rate in these schemes, it is necessary to create the condition under which the accelerating field must be much higher than the electric field decelerating the driving beam. Only in this case it would be possible to attain an effective gain in the accelerated particle energy. These conditions can be provided by making use of the field inhomogeneity in the cross section of the electrodynamic structure (radial beam separation) [5, 7-9], or by placing the beams at different resonances relative to the electromagnetic wave (two resonance acceleration schemes) $[7,10,12]$. Of all possible types of electrodynamic structures employed in this TBA group, the plasma waveguide (cavity) should be considered as most promising. In a plasma, the limiting electric field

strengths may reach great values (about $10^{6}$ to $10^{9} \mathrm{~V} / \mathrm{cm}$ ), and this allows, in principle, the 
attainment of a high charged-particle acculeration rate. In this case, the plasma, even being of a high density, remains "transparent" to accelerated particles.

In the plasma waveguide the condition of effective acceleration can be provided by making use of the inhomogeneous electric field distribution across the waveguide system. Fig. 1 shows a schematic of the radial structure $\Phi(r)$ of the longitudinal electric field component eigenmodes of circular plasma waveguide with an ideally conducting lateral surface, located in a strong longitudinal magnetic field [13]. It is seen that $\Phi(r)$ has its peak value on the waveguide axis $r=0$ and vanished on the lateral surface of the waveguide $r=r_{0}$. Hence, the efficient acceleration of charged particles requires that the driving beam should be injected into the region, where the electric field strength is minimum (near the lateral surface), and the accelerated beam - into the region, where the electric intensity reaches its peak value (close to the plasma system axis).

The main aim of this work is to demonstrate by theory the possibility of realizing effectively the TBA scheme in the plasma waveguide. The physical model of the TBA scheme under study is formulated in Sec. 2. Here we also give a set of equations describing the excitation of RF fields by a high-current REB and the acceleration of driven beam electrons. Section 3 presents the results of the linear theory of plasma wave amplification by the driving beam. The range of system parameters, at which the plasma-beam instability develops, is defined. Results of numerical simulation of the TBA scheme under study are presented in Sec. 4. The same section gives the description of the dynamics of accelerated particle bunching in the high-current REBexcited field. Estimates are given for the accelerating field intensities in the plasma and electron acceleration rates. Section 5 comprises the discussion of the results obtained and conclusions.

\section{The physical model and its basic equations}

The scheme of the two-beam charged particle acceleration considered in this paper is shown in Fig. 2. Here, the straight tubular beams, both driving and driven, with the radii $r_{1}$ and 
$r_{2}\left(r_{2} / r_{1}<1\right)$ move along the axis of a circular metallic waveguide. The waveguide is filled with a homogeneous collisionless "cold" plasma of density $n_{p}$.

The physical model adopted here describes the process of stationary amplification of plasma waves in the given electrodynamic structure and the acceleration of driven beam electrons by these waves. The model includes the following assumptions.

1. The beams have small transverse dimensions, so their stratification can be neglected.

2. The waveguide is in the strong longitudinal magnetic field $\vec{H}_{0} \| \vec{e}_{2}$, hence, the motion of electrons of the both beams and of the plasma is possible only in longitudinal direction (motion is one-dimensional).

3. The driven beam current is low in comparison with the driving one, therefore we shall ignore its effect on the process of field excitation (accelerated charged particles are moving in the given electromagnetic field).

4. The plasma is considered to be a linear medium, this being true provided that

$$
\tilde{v} / v_{p h}<1
$$

where $v_{p h}$ is the phase velocity of the wave; $\tilde{v}=e E_{2} / m \omega$ is the oscillation velocity of plasma electrons moving in the field $E_{z} ; \omega$ is the wave frequency; e,m are, respectively, the charge and mass of the electron. Plasma ions are assumed infinitely heavy and their contribution to plasma polarization is neglected.

5. All perturbations introduced by the driving beam are assumed to be azimuthally symmetric, so that the perturbed variables can be represented as $f=f(r, z) \exp (-i v), v=\omega t-h_{1} z$, where $t, z$ are the time and longitudinal coordinate, $h_{1}$ is the longitudinal wave number of the plasma-waveguide fundamental radial mode characterized by minimum root value of the zero-order Bessel function. The fundamental radial mode has the greatest phase velocity. We presume that at the given parameters of the system it is in resonance with the beam. This is illustrated by Fig. 3. which schematically shows the dispersion curves of the first 
three radial modes of the plasma waveguide. Note also that $f(r, z)$ is the amplitude, dependent on radial $(r)$ and longitudinal $(z)$ coordinates, which slowly varies in the longitudinal direction and satisfies the inequality

$$
|\partial \ln f(r, z) / \partial z| h_{1}^{-1}<<1
$$

6. We consider the plasma TM modes having the electric and magnetic field components $E_{2}, E_{r}, H_{\varphi}$ in the cylindrical coordinate frame. This arose from the fact that the straight beams may effectively interact only with the longitudinal component of the electric field $E_{z}$, which is inherent in the TM modes.

The original set of equations describing the excitation of the TM waves in the anisotropic plasma waveguide comprises Maxwell's equation written down in the cylindrical coordinates:

$$
\begin{gathered}
\frac{\partial E_{r}}{\partial z}-\frac{\partial E_{z}}{\partial r}=i \frac{\omega}{c} H_{\varphi}, \\
\frac{\partial H_{\varphi}}{\partial z}=i \frac{\omega}{c} E_{r}, \\
\frac{1}{r} \frac{\partial}{\partial r}\left(r H_{\varphi}\right)=-i \frac{\omega}{c} \varepsilon_{p} E_{z}+\frac{4 \pi}{c} j_{\omega},
\end{gathered}
$$

where $\varepsilon_{p}=1-\omega_{p}^{2} / \omega^{2}$ is the longitudinal component of the plasma permittivity,

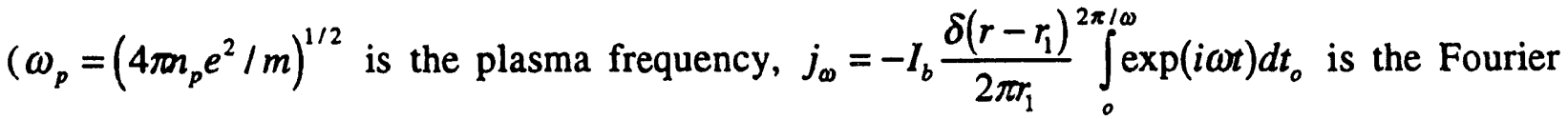
component of the driving REB current density at the frequency $\omega, I_{b}$ is the beam current, $t_{o}$ denotes initial times of electron arrival to the waveguide. On writing the expression for $j_{\omega}$ we have made use of the law of beam charge conservation.

According to the general theory of waveguide excitation [14], the expressions for the fields entering into (3)-(5) can be written as 


$$
\begin{gathered}
\vec{E}=\sum_{s=1}^{\infty}\left(C_{s} \vec{E}_{s}+C_{-s} \vec{E}_{-s}\right)+\frac{4 \pi j_{\omega} \vec{e}_{z}}{i \omega E_{p}} \\
\vec{H}=\sum_{s=1}^{\infty}\left(C_{s} \vec{H}_{s}+C_{-s} \vec{H}_{-s}\right)
\end{gathered}
$$

where $\vec{E}_{ \pm s}, \vec{H}_{ \pm s}$ are the fields of the eigenmodes of the plasma waveguide without the beam $\left(\vec{E}_{-s}=\vec{E}_{s}^{*}, \vec{H}_{-s}=-\vec{H}_{s}^{*}\right), C_{ \pm s}$ are their amplitudes. The summation in (6), (7) is made over all radial modes of the waveguide.

The eigenmodes amplitudes $C_{ \pm s}$ are subjected to the excitation equations

$$
\frac{d C}{d z} \pm s= \pm \frac{1}{N_{s}} \int_{s_{\perp}} j_{\omega} E_{\mp_{s z}} d s_{\perp}
$$

where $S_{\perp}$ is the waveguide cross section, $N_{s}=\frac{C}{4 \pi} \int_{S_{\perp}}\left\{\left[\vec{E}_{s} \times \vec{H}_{-s}\right]-\left[\vec{E}_{-s} \times \vec{H}_{s}\right]\right\} \vec{e}_{2} d S_{\perp}$ is the norm of the $S$-th radial mode.

As is known [13], eigenfunctions of the axially symmetric TM modes of the anisotropic plasma waveguide satisfy the expressions

$$
\begin{gathered}
\vec{E}_{s}=-\frac{i h_{s} \varepsilon_{p}}{k_{s}} J_{1}\left(k_{s} r\right) \exp \left(i h_{s} z\right) \vec{e}_{r}+J_{o}\left(k_{s} r\right) \exp \left(i h_{s} r\right) \vec{e}_{z}, \\
\vec{H}_{s}=-\frac{i \omega \varepsilon_{p}}{C k_{s}} J_{1}\left(k_{s} r\right) \exp \left(i h_{s} z\right) \vec{e}_{\varphi}
\end{gathered}
$$

where $J_{0}, J_{1}$ are the zero and first-rder Bessel functions, $h_{s}$ is the longitudinal wave number corresponding to the $S$ - th radial mode; $k_{s}=\left(\omega^{2} / C^{2}-h_{s}^{2}\right)^{1 / 2} \varepsilon_{p}^{1 / 2}, k_{s}=\lambda_{s} / r_{o}, \lambda_{s}$ are the roots of the zero-order Bessel function $J_{o}\left(\lambda_{s}\right)=0$. In view of expressions (9), (10), the equations for $C_{s}$ can essentially be simplified

$$
\frac{d C}{d z} \pm S=\mp \frac{I_{b} J_{o}\left(\lambda_{s} r_{1} / r_{o}\right)}{\pi N_{S}} \exp \left[i\left(h_{1} \mp h_{S}\right) z\right] \rho
$$


where $N_{s}=-\frac{1}{2} r_{o}^{4} \varepsilon_{p}^{2} J_{1}^{2}\left(\lambda_{s}\right) h_{s} \omega \lambda_{s}^{-2}, \rho=\int_{0}^{2 \pi} \exp (i \vartheta) d \vartheta_{o}, \vartheta_{o}=\omega t_{0}$.

We now derive the explicit expression for the longitudinal component of the electric field acting on the beams electrons. To do this, we separate from the sum in (6) the component corresponding to the fundamental radial mode $S=1$ at the frequency $\omega$, which is in resonance with the beam and whose amplitude $C_{1}$ obeys the excitation equation.

$$
\frac{d C_{1}}{d z}=-\frac{I_{b} J_{o}\left(\lambda_{1} r_{1} / r_{o}\right) \rho}{\pi N_{1}}
$$

The approximate values of nonresonance amplitudes $C_{ \pm S}$ can be obtained from (11) through integration by parts

$$
C_{ \pm S}=\mp \frac{I_{b} J_{o}\left(\lambda_{S} r_{1} / r_{o}\right)}{\pi N_{S}}\left[\frac{\rho}{i\left(h_{1} \mp h_{S}\right)}+\frac{d \rho / d z}{\left(h_{1} \mp h_{S}\right)^{2}}\right] \exp \left[i\left(h_{1} \mp h_{S}\right) z\right]
$$

Note that by assumption (2) the second term in (13) is small as compared with the first one. Substituting (13) into (6) and using the expansion of the $\delta$-function, entering into the expression for $j_{\omega}$, in series in the Bessel functions

$$
\frac{\delta\left(r-r_{1}\right)}{r_{1}}=\sum_{s=1}^{\infty} \frac{2 J_{o}\left(\lambda_{s} r / r_{o}\right) J_{o}\left(\lambda_{s} r_{1} / r_{o}\right)}{J_{1}^{2}\left(\lambda_{s}\right) r_{o}^{2}}
$$

we obtain the following expressions for the longitudinal component of the electric field

$$
\begin{aligned}
& E_{z}(r, z)= \\
& {\left[C_{1} J_{o}\left(\lambda_{1} r / r_{o}\right)-i \frac{4 I_{b} \omega m c^{3}(1+\sigma) J_{o}\left(\lambda_{1} r / r_{o}\right) J_{o}\left(\lambda_{1} r_{1} / r_{o}\right) \rho \Pi(r)}{I_{A} \lambda_{1}^{2} e \gamma_{o}^{2} v_{0}^{2} J_{1}^{2}\left(\lambda_{1}\right)}+\frac{8 I_{b} m c^{3} h_{1} \wedge(r)(d \rho / d z)}{I_{A} \omega e}\right]} \\
& \exp \left(i h_{1} z\right)
\end{aligned}
$$


where $I_{A}=m c^{3} / e \cong 17 k A, \gamma_{0}=\left(1-\beta_{o}^{2}\right)$ is the relativistic beam, $\beta_{o}=V_{o} / C, V_{0}$ being the initial velocity of the driving REB, $\sigma=2 \gamma_{0}^{2} \Delta, \Delta=h_{1} V_{0} / \omega-1$ is the detuning parameter.

$$
\begin{gathered}
\Pi(r)=1-\frac{J_{1}^{2}\left(\lambda_{1}\right) \lambda_{1}^{2}}{J_{0}\left(\lambda_{1} r / r_{0}\right) J_{0}\left(\lambda_{1} r_{1} / r_{o}\right)} \sum_{s=2}^{\infty} \frac{J_{0}\left(\lambda_{s} r / r_{o}\right) J_{o}\left(\lambda_{s} r_{1} / r_{o}\right)}{J_{1}^{2}\left(\lambda_{s}\right)\left(\lambda_{s}^{2}-\lambda_{1}^{2}\right)} \\
\wedge(r)=\sum \frac{J_{0}\left(\lambda_{s} r / r_{0}\right) J_{0}\left(\lambda_{s} r_{1} / r_{o}\right) \lambda_{s}^{2}}{J_{1}^{2}\left(\lambda_{s}\right)\left(\lambda_{s}^{2}-\lambda_{1}^{2}\right)^{2}}
\end{gathered}
$$

The first term in (15) represents the field of the wave being in resonance with the beam, the second and third terms stand for the field of the RF space charge of the high-current REB.

Having defined the expression for the field $E_{z}$, one can obtain a self-consistent set of equations describing the anisotropic plasma waveguide excitation by a high-current REB. To this end, the excitation equation (12) should be complemented with the equations of motion for the phase $\vartheta=\omega t-h_{1} z$ and the momentum $p$ of driving beam electrons moving in the field $E_{z}$ :

$$
\begin{gathered}
\frac{d p}{d z}=-\frac{e\left(p^{2}+m^{2} c^{2}\right)^{1 / 2}}{p c} \operatorname{Re}\left[E_{2}\left(r=r_{1}\right) \exp (-i \vartheta)\right] \\
\frac{d \vartheta}{d z}=-\frac{\omega\left(p^{2}+m^{2} c^{2}\right)^{1 / 2}}{p c}-h_{1}
\end{gathered}
$$

For further analysis, it is convenient to represent Eqs. (12), (18), (19) in the dimensionless form

$$
\begin{gathered}
\frac{d A}{d \xi}=\frac{v_{b}}{2 \pi}(1+\sigma)^{2} \rho \\
\frac{d q}{d \xi}=-\frac{\left(1+q^{2}\right)^{1 / 2}}{q} \operatorname{Re}\left\{\left[A(\xi)+F_{1} \Pi_{1} \rho+F_{2} \wedge_{1} d \rho / d \xi\right] \exp (-i \vartheta)\right\} \\
\frac{d \vartheta}{d \xi}=\beta_{o} \frac{\left(1+q^{2}\right)^{1 / 2}}{q}-1-\Delta,
\end{gathered}
$$




$$
\frac{d \vartheta}{d \xi}=\beta_{o} \frac{\left(1+q^{2}\right)^{1 / 2}}{q}-1-\Delta
$$

where $A=\frac{C_{1} J_{o}\left(\lambda_{1} r_{1} / r_{o}\right) e V_{o}}{m c^{2} \omega}, F_{1}=-2 i\left(v_{b} / 2 \pi\right) \gamma_{o}^{2}(1+\sigma), F_{2}=4\left(v_{b} / 2 \pi\right) \gamma_{o}^{4} \lambda_{1}^{2}$,

$\frac{v_{b}}{2 \pi}=\frac{C_{1} J_{0}^{2}\left(\lambda_{1} r_{1} / r_{o}\right)}{\pi I_{A} \lambda_{1}^{2} J_{1}^{2}\left(\lambda_{1}\right) \gamma_{o}^{4} \beta_{o}}, \Pi_{1}=\Pi\left(r=r_{1}\right), \Lambda_{1}=\Lambda\left(r=r_{1}\right) J_{1}^{2}\left(\lambda_{1}\right) / J_{o}^{2}\left(\lambda_{1} r_{1} / r_{o}\right), \xi=\omega z / v_{o}$,

$q=p / m c$.

The equations describing the motion of driven beam electrons in the field $E_{z}$ can be written

as

$$
\begin{gathered}
\frac{d q^{a c c}}{d \xi}=-\frac{\left[1+\left(q^{a c c}\right)^{2}\right]^{1 / 2}}{q^{a c c}} \operatorname{Re}\left\{\left[A(\xi)+F_{1} \Pi_{2} \rho\right]+F_{2} \Lambda_{2} K^{-1} d \rho / d \xi\right\} \times\left[\exp \left(-i \vartheta^{a c c}\right)\right] K, \\
\frac{d \vartheta^{a c c}}{d \xi}=\beta_{o} \frac{\left[1+\left(q^{a c c}\right)^{2}\right]^{1 / 2}}{q^{a c c}}-1-\Delta,
\end{gathered}
$$

where $q^{\text {acc }}=p^{\text {acc }} / m c, p^{\text {acc }}$ is the momentum of the driven beam particles, $\vartheta^{a c c}$ is the phase of the driven beam electrons, $\Pi_{2}=\Pi\left(r=r_{2}\right), \quad \Lambda_{2}=\Lambda\left(r=r_{2}\right) J_{1}^{2}\left(\lambda_{1}\right) / J_{o}^{2}\left(\lambda_{1} r_{1} / r o\right)$, $K=J_{0}\left(\lambda_{1} r_{2} / r_{0}\right) / J_{0}\left(\lambda_{1} r_{1} / r_{0}\right)$ is the parameter defining the ration of the fundamental radial mode amplitude in the region of the driven beam $\left(r=r_{2}\right)$ to that in the region of the driving beam $\left(r=r_{1}\right)$. The values of this ratio essentially determines the efficiency of driven REB acceleration.

$\Pi_{1,2}$ and $\Lambda_{1,2}$, entering into the equations of motion (21), (23) were calculated by summing the series (16), (17). The values of these parameters depend on the ratios of the driving and driven beams radii to the waveguide radius. Further on, we consider the radius of the driven REB to be fixed $r_{2} / r_{0}=0,1$. In view of this, Fig. 4 shows $\Pi_{1,2}$ and $\Lambda_{1,2}$ as functions of ratio of the driving $\mathrm{RE}: \mathrm{B}$ radius to the waveguide radius. 
Equations (20) - (24) must be complemented with the boundary conditions

$$
\begin{aligned}
& A(\xi=0)=A_{o}, q(\xi=0)=q_{o}, q^{a c c}(\xi=0)=q_{o}^{a c c} \\
& \vartheta(\xi=0)=\vartheta_{o} \in[0,2 \pi], \vartheta^{a c c}(\xi=0)=\vartheta_{o}^{a c c} \in[0,2 \pi]
\end{aligned}
$$

Note that Eqs. (20)-(24) comprise the integral representing the law of energy conservation

$$
\begin{aligned}
& \left(v_{b} / 2 \pi\right) \int_{0}^{2 \pi}\left(1+q^{2}\right)^{1 / 2} d \vartheta_{o}+0.5|A|^{2}(1+\sigma)^{-2} \\
& +2\left(v_{b} / 2 \pi\right)^{2} \gamma_{0}^{4} \lambda_{1}^{2} \Lambda_{1} \times\left[\left(\int_{0}^{2 \pi} \cos \vartheta d \vartheta_{o}\right)^{2}+\left(\int_{0}^{2 \pi} \sin \vartheta d \vartheta_{0}\right)^{2}\right]=\text { const }
\end{aligned}
$$

where the first term defines the energy contribution of the beam, and the second and third terms define the contributions from the amplified resonance wave and nonresonance harmonics of the field to the total energy balance of the electrodynamic system. From (26) we obtain the expression giving the efficiency of driving REB energy transformation into the field energy:

$$
\begin{aligned}
\eta= & \frac{1}{2 \pi} \int_{0}^{2 \pi} \frac{\gamma_{0}-\left(1+q^{2}\right)^{1 / 2}}{\gamma_{0}-1} d \vartheta_{o}=\eta_{1}+\eta_{2}=\frac{|A|^{2}-\left|A_{o}\right|^{2}}{2 v_{b}(1+\sigma)^{2}\left(\gamma_{0}-1\right)} \\
& +\frac{v_{b} \gamma_{0}^{4} \lambda_{1} \Lambda_{1}}{2 \pi^{2}\left(\gamma_{0}-1\right)} \times\left[\left(\int_{0}^{2 \pi} \cos \vartheta d \vartheta_{o}\right)^{2}+\left(\int_{0}^{2 \pi} \sin \vartheta d \vartheta_{o}\right)^{2}\right]
\end{aligned}
$$

where $\eta$ is the relative fraction of total beam kinetic energy losses, $\eta_{1}$ and $\eta_{2}$ are the relative fractions of beam energy losses by excitation of the resonance plasma wave and nonresonance harmonics of the plasma waveguide. 


\section{The linear stage of plasma wave amplification}

We now consider in brief the linear stage of anisotropic plasma waveguide excitation by a high-current REB. At the initial stage of instability, when the amplitudes of RF fields excited in the plasma waveguide are small, the self-consistent set of Eqs. (20)-(22) can be linearized as

$$
\begin{gathered}
\frac{d A}{d \xi}=\left(v_{b} / 2 \pi\right)(1+\sigma)^{2} \cdot \tilde{\rho}, \\
\frac{d \tilde{q}}{d \xi}=-\frac{1}{2 \beta_{o}} \operatorname{Re}\left\{\left[A(\xi)+F_{1} \Pi_{1} \tilde{\rho}+F_{2} \Lambda_{1} d \tilde{\rho} / d \xi\right] \exp \left(-i \vartheta_{o}+i \Delta\right)\right\}, \\
\frac{d \tilde{\vartheta}}{d \xi}=-\frac{\tilde{q}}{\beta_{o}^{2} \gamma_{o}^{3}}-\Delta,
\end{gathered}
$$

where $\tilde{\rho}=i \int_{0}^{2 \pi} \tilde{\vartheta} \exp \left[i\left(\vartheta_{o}-\Delta \xi\right)\right] d \vartheta_{o} ; \tilde{\vartheta}, \tilde{q}$ are the perturbed quantities relative to their equilibrium values $\vartheta_{o}, q_{o}$. Writing the perturbed $\tilde{\rho}, \tilde{\vartheta}, \tilde{q}$ in term of A, we reduce Eqs. (28)-(30) to the equation

$$
\frac{d^{3} A}{d \xi^{3}}+i\left(2 \Delta-a_{1}\right) \frac{d^{2} A}{d \xi^{2}}-\left(\Delta^{2}+a_{2}\right) \frac{d A}{d \xi}-i a_{3} A=0
$$

wh er e $\quad a_{1}=4 \pi \beta_{o}^{-2} \gamma_{o} \lambda_{1}^{2} \Lambda_{1}\left(v_{b} / 2 \pi\right), \quad a_{2}=2 \pi \beta_{o}^{-2} \gamma_{o}^{-1}(1+\sigma) \Pi_{1}\left(v_{b} / 2 \pi\right)$, $a_{3}=\pi \beta_{o}^{-2} \gamma_{o}^{-3}(1+\sigma)^{2}\left(v_{b} / 2 \pi\right)$.

Representing the solution of (31) as $A \sim \exp (-i \Gamma \xi)$, we come to the following dispersion equation

$$
\Gamma\left[(\Gamma+\Delta)^{2}-a_{1} \Gamma+a_{2}\right]+a_{3}=0
$$

which enables us to determine the range of the system parameters, at which the plasma-beam instability develops, as well as to find out the peak value of the space increment $\operatorname{Im} \Gamma$. 
Equation (32) can easily be solved by the Cardano formulae. The solutions were found for different values of the driving REB current, radius and detuning $\Delta$. The beam energy was taken to be $\gamma_{0}=5$. Figures 5-7 show $\operatorname{Im} \Gamma$ versus $\Delta$ for different $I_{b}$ and $r_{1} / r_{0}$ values. It is seen from these figures that the region of detunings, where the increment is non-zero, grows with $I_{b}$ towards positive $\Delta$ values. The increment reaches its peak value somewhere in the middle of the region of detuning, at which the instability in the plasma-beam system takes place. This instability regime corresponds to the Raman regime of plasma wave excitation.

As the driving REB current decreases the peak value of the space increments shifts to the zero detuning region. For low-current beams $\left(I_{b} / I_{A} \ll 1\right)$ the space increment reaches its highest value at $\Delta=0$ (Compton regime of wave excitation). In this case, the field of the RF space charge can be neglected. Then from (32) and for $\Delta=0$ we obtain the expression for the greatest space increment

$$
\operatorname{Im} \Gamma=\frac{3^{1 / 2}}{2} a_{3}^{1 / 3}
$$

Note that this expression is well known in the theory of low-current amplifiers of the Cherenkov type.

\section{Results of numerical simulations}

The scheme of two-beam acceleration of charged particles in an anisotropic plasma waveguide was studied by numerical simulation of the set of Eqs. (20)-(24). We shall first analyze the results of numerical calculations describing the process of fields excitation by the driving REB (Eqs.(20)-(22)). These calculations were carried out for different driving REB current values. The initial energy of the beam as well as its average radius were chosen to be $\gamma_{0}=5 ; r_{1}=0.65 r_{0}$ and $0.81 r_{o}$. The $\Delta$ value was chosen so that for the given REB parameters the space increment 
should be maximum (optimization of the system by the space increment, Figs. 5 to 7 ). The boundary conditions for the amplified wave were set to be $\operatorname{Re} A_{o}=5.10^{-3} ; \operatorname{Im} A_{o}=0$.

It should be mentioned that the condition of plasma linearity imposes a restriction on the current value $I_{b}$. Really, with increasing $I_{b}$ the amplitude of the excited field may grow to such values at which a portion of driving beam electrons full gives away all its energy to the wave field and stops. In this case, of great importance may become the effects associated with plasma nonlinearity, including trapping of plasma particles by the wave field. Below we shall consider only those $I_{b}$ values which satisfy the condition $I_{b}<I_{b}^{*}$, where $I_{b}^{*}$ is the limiting current value at which the beam particle do not stop as yet. The accuracy of numerical calculations was controlled by means of the integral (26) expressing the law of energy conservation.

The process of RF field excitation is analyzed for $r_{1}=0.65 r_{o}(K=2)$. To show the special features of high-current effects in the plasma-beam interaction we put the $I_{b}$ value close to the $I_{b}^{*}$ ( $I_{b}=17 \mathrm{kA}$ for the optimum detuning $\Delta=0.03$ ). Strictly speaking, even at $I_{b}=17 \mathrm{kA}$ there may appear the effects associated with plasma nonlinearity close to the waveguide axis, where the electric field intensity is highest. The analysis of these effects requires additional thorough studies, which are beyond the scope of this work.

Fig. 8 show the amplitude $|A(\xi)|$ and the field phase $\operatorname{Arg} A(z)$ of the wave, being in resonance with the beam, versus the longitudinal coordinate $\xi$. For comparison, the same figure shows similar dependences calculated without taking into account the RF space charge $\left(\Lambda_{1}=\Pi_{1}=0\right)$. It is seen that at the linear stage of amplification the resonance wave amplitude exponentially grows. Then the exponential growth is followed by regular space oscillations due to phase oscillations of bunches in the wave field[15]. It follows from the dependencies that the RF space charge furces lead to the decrease in the peak-to peak amplitude oscillations. This is a consequence of a partial breakup of beam bunches and phase mixing of particles. The process of bunch breakup is illustrated by Fig. 9, which shows phase portraits of driving REB particles at different $\xi$ values. 
It is of importance to note that for the system parameters considered the main channel of high-current beam energy losses is the excitation of a synchronous wave. This is clearly evident from Fig. 10 which shows the $\xi$-dependences of the relative part of total kinetic beam energy losses $\eta$, relative energy fluxes of the synchronous plasma wave $\eta_{1}$, and nonresonance harmonics of the plasma waveguide $\eta_{2}$, in the driving beam region $\left(r=r_{1}\right)$. The peak of total losses is observed at the $\xi=150$, making about $\eta \approx 28 \%$. Nearly at the same point the losses by the resonance plasma wave amplification are also the greatest, $\eta_{1}=24 \%$. The maximum losses by the excitation of nonresonance field harmonics, contributing to the resultant value of the RF space charge field, do not exceed $\eta_{2} \approx 4 \%$. However, this does not mean that the RF space charge forces of the high-current REB weakly affect the particle acceleration. In fact, at different points of the interaction space $r=r_{1}$ (deriving REB region) and $r=r_{2}$ (driven REB region) the RF space charge field amplitude values may essentially differ between themselves (compare $r$-dependencies of $\Lambda_{1,2}$ and $\Pi_{1,2}$ ). Therefore, to have a total picture of process of charged particle acceleration, it is necessary to determine the contribution of the space charge field to the resultant electric field intensity in the $r=r_{2}$ region.

Let us consider the excitation of the accelerating field acting on the electrons of the driven beam $\left(r=r_{2}\right)$. Figure 11 shows the $\xi$-dependences of the amplitude $|\tilde{A}|$ and phase $\operatorname{Arg} \tilde{A}(\xi)$ of the field in the driven REB region $\tilde{A}(\xi)=K\left[A(\xi)+F_{1} \Pi_{2} \rho+F_{2} \Lambda_{2} K^{-1} d \rho / d \xi\right]$. It is evident from the figure that with a growing longitudinal component $\xi$ the exponential growth of the amplitude changes into irregular space oscillations. In this case the greatest field amplitude $\left|\tilde{A}_{\max }\right| \approx 0.52$ is attained at $\xi \approx 142$ (first maximum region). At $\xi>142$ the amplitude abruptly falls off and its value is not above $|\tilde{A}| \approx 0.28$. The RF space charge field of the high-current REB causes an appreciable increase in the accelerating field amplitude $|\tilde{A}(\xi)|$. Yor example, at $\xi=142$ the $|\tilde{A}|$ value is approximately by factor of 2.2 greater than the resonance wave amplitude value in the driven REB region $|A| K=0.24$ (see Figs. 8a and 11a). This is rather advantageous for employing high-current REBs to accelerate charged particles. Note that the above-described behavior of the 
field amplitude $|\tilde{A}(\xi)|$ qualitatively corresponds to the results of the numerical analysis of field excitation carried out for other high-current beam parameters.

We now consider the dynamics of charged particle acceleration described by the equations of motion (23), (24). The acceleration process will be analyzed for the same driving REB parameters chosen above for the analysis of RF field excitation, i.e., $r_{1}=0.65 r_{o}, I_{b}=I_{A}$, $\Delta=0.03$. The initial driven REB energy is taken equal to the initial energy of the driving REB, $\gamma_{0}^{a c c}=\gamma_{0}=5$. At given beam parameters, it is of importance to determine the optimum length 1 of the accelerating structure, over which the most effective gain in the electron energy is attained. For this purpose we have plotted the highest momentum of the driven beam particles $q_{\max }^{a c c}$ versus $\xi$ (Fig. 12). It is evident from the plot that at $\xi \leq 170$ the momentum grows reaching $q_{\max }^{a c c}=13$, this is approximately by 2.6 times greater than the initial electron momentum $q_{o}^{a c c}=\left[\left(\gamma_{o}^{a c c}\right)^{2}-1\right]^{1 / 2}=4.89$. The distance over which this momentum value is attained is comparable with the distance to the first maximum of the accelerating field amplitude $\xi=142$ (see Fig. 11a). Then $q_{\max }^{a c c}$ monotonically decreases. The cessation of the process of acceleration is due to the change in the polarity of the particle-accelerating field. As a result, the particle being in the accelerating phase of the field and having the highest momentum at $\xi=170$ turns out to be in the decelerating phase, and its energy starts to decrease. With increasing $\xi$, the process $q_{\max }^{a c c}$ of decrease and growth recurs. However, the $q_{\max }^{a c c}$ value does not exceed 13 . This is due to the reduction at $\xi>142$ of the particle-accelerating field amplitude (see Fig. 11a). So, for the parameters considered the length $1 \approx 165$ to 170 appears optimum from the viewpoint of attaining the highest particle energy at the exit.

The change of the beam parameters may lead to the corresponding change in the optimum length of the accelerating structure. Therefore, for each set of the parameters of a TBA scheme the optimization in 1 should be carried out, and this was done in the present work. It has been established that the most efficient process of acceleration takes place over the distance which approximately corresponds to the distance to the first maximum of the field amplitude in the region of the driven $\operatorname{REB}|\tilde{A}(\xi)|$. 
Let us consider the dynamics of accelerated particle bunch formation. In compliance with the above given results, we put $l=168$. Figures 13 and 14 show the phase planes of the driven beam particles $\left(q^{\text {acc }}, \varphi^{\text {acc }}=\operatorname{Arg} \tilde{A}(\xi)-\vartheta^{\text {acc }}\right)$ and the dependencies of electron moments on the initial phases $\vartheta_{o}^{a c c}$ at different points of the interaction space. It is seen from the figures that even at the beginning of the accelerating structure $\xi<84$ there takes place the formation of accelerated and decelerated particle bunches. For this part of the interaction space the field amplitude $|\tilde{A}(\xi)|$ is small (see Fig. 11a). Therefore, the particle momentum values are close to $q_{o}^{\text {acc }}$. In this case, as it follows from (24), the phases $\vartheta^{\text {acc }}(\xi)$ change with increasing $\xi$ practically by the linear law $\vartheta^{a c c} \sim-\Delta \xi$. Note that this dependence causes the change in the polarity of the field acting on the particles. Really, as the length of the interaction space increases, the difference $\vartheta_{0}^{a c c}-\vartheta^{a c c}$ tends to $\pi$. At the same time, the $\operatorname{Arg} \tilde{A}(\xi)$ value changes insignificantly over the accelerating structure length (see Fig. 11b). Therefore, at $\vartheta_{o}^{a c c}-\vartheta^{a c c} \rightarrow \pi$ the polarity of the particle-accelerating field changes the sign: $\varphi^{a c c}(\xi=0)-\varphi^{a c c}(\xi=\xi)=\pi$, where $\xi$ is a certain longitudinal coordinate value, at which the phase $\varphi^{a c c}$ advance is $\pi$. As calculations show, for the majority of particles the $\xi$ value ranges between 100 and 120 . At $\xi=\xi$ the particles belonging to the accelerated bunch start to decelerate. As to previously decelerated particles, some part of them is trapped into the acceleration regime which persists to the end of the interaction space (Figs. 13, 14). At the exit, the accelerated particles make up nearly $10 \%$ of the total number of driven beam particles.

To represent the described process of acceleration more obviously, Fig. 15, shows the momentum of the accelerated particle with the initial phase $\vartheta_{o}^{\text {acc }}=0.47$ as a function of the longitudinal coordinate (curve 1). It is clearly seen from the figure that the most effective acceleration is realized only over a small part of the plasma waveguide, which is adjacent to the output end of the latter $130 \leq \xi \leq 168$. As demonstrated above, the non uniform energy gain is due to a rather rapid change of $\vartheta^{\text {acc }}$ at the beginning of the accelerating structure due to the finite detuning value $\left(\vartheta^{a c c} \sim \Delta \xi\right)$. To attain a uniform energy gain by accelerated particles, it is necessary that the initial velocity of the driven REB, $v_{o}^{\text {acc }}$, should be equal to the phase velocity of the wave $v_{p h}=\omega / h_{1}$ (condition of the exact Cherenkov resonance). At $\Delta=0 / 03$ the initial energy 
$\gamma_{o}^{a c c}=3.24$ corresponds to this condition. For this energy the optimum plasma waveguide length is approximately 152 . Fig. 16 shows $q^{a c c}$ versus $\vartheta_{o}^{\text {acc }}$ for different $\xi$ values. It is seen that the energy gain by the accelerated particles takes place more uniformly than in the $\gamma_{o}^{a c c}=5$ case. This is also illustrated by the $\xi$-dependence of the momentum of the accelerated particle with the initial phase $\vartheta_{o}^{a c c}=4.08$ (see Fig. 15, curve 2). At the exit, the highest accelerated particle energy is nearly by 3.5 times greater than the primary energy of the particles. So, the uniform energy gain at $\gamma_{o}^{a c c}=3.24$ also results in the higher charged particle acceleration rate than in the $\gamma_{o}^{a c c}=5$ case.

We now give the estimates for the greatest amplitude of the electric field longitudinal component in the region of the driven beam and for the electron acceleration rate. The plasma density is put to be $n_{p}=10^{14} \mathrm{~cm}^{-1}$. For the above given parameters of the driving REB the greatest amplitude of the accelerating field is (see Fig. 11a): $\left|E_{\max }\right|=\left|\tilde{A}_{\max }\right| m c^{2} \omega / e v_{o}=0.52 m c^{2} \omega / e v_{o}$. To estimate $\omega$, we resort to the condition of the Cherenkov resonance of the driving REB with the fundamental radial mode [13]: $\omega=\gamma_{o} \nu_{o}\left[\left(\omega_{p} / \nabla_{o} \gamma_{0}\right)^{2}-\left(\lambda_{1} / r_{o}\right)^{2}\right]^{1 / 2}$. Hence, on condition that $\left(\omega_{p} / v_{o} \gamma_{o}\right)>\left(\lambda_{1} / r_{o}\right)$ we obtain $\omega \approx \omega_{p}$. In view of this, the greatest accelerating field amplitude is $\left|E_{\max }\right|=500 \mathrm{Mv} / \mathrm{m}$. First we estimate the electron acceleration rate for the case of $\gamma_{o}^{a c c}=3.24$. The plasma waveguide length is $L=152 v_{o} / \omega_{p} \approx 7.9 \mathrm{~cm}$. As it follows from Figs. 15, 16, the greatest energy gain over this length is $\Delta W=m c^{2}\left(\gamma^{a c c}-\gamma_{o}^{a c c}\right)=4.1 \mathrm{MeV}, \gamma^{a c c}=\left[1+\left(q^{a c c}\right)^{2}\right]^{1 / 2}$. As a result, we get $T=\Delta W / L=52 \mathrm{Mev} / \mathrm{m}$. At $\gamma_{0}^{\text {acc }}=5$ we have the following parameters of the TBA scheme: $L=8.7 \mathrm{~cm}, \Delta W=4 \mathrm{Mev}, T=46,5 \mathrm{Mev} / \mathrm{m}$. It should be noted that the inhomogeneous electric field distribution along the interaction space (Fig. 11a) and the phase variation $\varphi^{\text {acc }}$ during charged particle acceleration considerably restrict the $T$ value. Therefore, as is seen from given estimates, there is a great difference between $\left|E_{\max }\right|$ and the acceleration rate $T$.

The process of charged particle acceleration was numerically analyzed for different driving REB current values at $r_{1}=0.65 r_{o}$ and $\gamma_{o}=5$. Table 1 lists the values of the main parameters of the TBA scheme under study, resulting from this analysis at $n_{p}=10^{14} \mathrm{~cm}^{-3}$.

The increase in the driving REB radius $r_{1}=0.81 r_{o}$ does not qualitatively change the above processes of RF field excitation and driven-beam particle acceleration. Note that as the ratio $r_{1} / r_{o}$ 
grows the transformation coefficient increases, $K=3.86$ and the coupling between the highcurrent REB and the resonance wave reduces $v_{b} / 2 \pi \sim J_{o}^{2}\left(\lambda_{1} r_{1} / r_{o}\right)$ (plasma waves are volumetric in plasma waveguide). Therefore, to attain high accelerating field intensities, it is necessary to increase the driving beam current. This can be seen from Table 2, which gives the main characteristics of the TBA scheme in the plasma waveguide at $r_{1}=0.81 r_{o}$ and $n_{p}=10^{14} \mathrm{~cm}^{-3}$.

To conclude this section, we shall discuss the problem of increasing the amount of particles in the accelerated bunch. This problem is of importance because it is closely connected with the problem of increasing the intensities of accelerated beams. As the present results indicate, the accelerated particles at the exit of the system amount to no more than $10 \%$ of the total number of driven beam particles (Figs. 14, 16). This percentage can essentially be increased by prebunching the particles in the phase $\vartheta_{o}^{\text {acc }}$. It is seen, for example, from Fig. 14d that the driven beam particles should be located in a narrow phase interval $\Delta \vartheta_{o}^{\text {acc }}$ in the vicinity of $\vartheta_{o}^{\text {acc }}=0.4$. In this case, all particles may be trapped into the acceleration regime and reach the energy $\gamma^{a c c} \approx 13$. So, we come to the necessity of using prebunched charged particles as a driven REB. One of the principal characteristics of the accelerated charged particle bunches is the relative particle energy scattering at the exit of the electrodynamic structure:

$\mu=\left\{\frac{1}{N} \sum_{i=1}^{N}\left[\gamma_{i}^{a c c}(\xi=1)-\bar{\gamma}^{a c c}(\xi=1)\right]^{2}\right\}^{1 / 2} / \bar{\gamma}^{a c c}(\xi=1)$, where $\bar{\gamma}^{\text {acc }}(\xi=1)=\frac{1}{N} \sum_{i=1}^{N} \gamma_{i}^{a c c}(\xi=1)$,

$N$

33being the amount of particles in the bunch. The parameter $\mu$ determines to a large measure the quality of the accelerated bunch. Figure 17 shows $\mu$ and $\bar{\gamma}^{\text {acc }}$ versus the phase interval $\Delta \vartheta^{\text {acc }}$ for the following parameters of the system: $r_{1} / r=0.65, I_{b}=I_{A}, \gamma_{o}^{a c c}=5,1=168$. It is evident that the TBA scheme under study can provide for accelerated bunches with a low energy scattering $\mu=3-7 \%$. This allows us to conclude that phase-grouped charged particle bunches can efficiently be used as a driven beam. 


\section{Conclusion}

The results presented above from the theoretical study of the concept of a two-beam acceleration in a plasma waveguide can be summarized as follows.

1. The set of nonlinear equations has been derived in the framework of the stationary model to describe the process of accelerating field excitation by a high-current driving beam and the acceleration of driven beam electrons by these fields.

2. The linear stage of the driving beam instability in the plasma waveguide has been investigated. The system parameters at which the plasma-beam instability develops, are established. The greatest space increment values are found for different driving REB current.

3. Numerical methods have been used to investigate the accelerating-field excitation by a highcurrent REB. It is found that the process of field excitation is influenced by the RF space charge field causes a substantial increase of the field intensity in the region of the driven beam.

4. The dynamics of accelerated particle bunching is described. Estimates are given for the highest accelerating-field amplitude acceleration rate and the optimum plasma waveguide length at different external parameters of the both (driving and driven) beams.

5. A possibility of using preliminary phase-grouped electron bunches as a driven beam for the purpose of increasing the amount of particles in the accelerated bunch is discussed. 


\section{References}

1. T. H. Martin, Design and Performance of the Sandia Laboratories Hermes II Flash X-ray Generator // IEEE Trans.Nucl.Sci. 1969. V.NS-16. N3. P.59-64

2. B. Bernstein, J. Smith. "Aurora", an Electron Accelerator // IEEE_Trans. Nucl. Sci. 1973. V.NS-20. N3. P.294-300

3. A. N. Didenko, V. P. Grigor'ev, Yu. P. Usov. High-Power Electron Beams and their Applications. Moscow. Atomizdat Publ. 1977. 277 p. (in Russ.)

4. A. M.Sessler, The Free Electron Laser as a Power for a High-Gradient Accelerating Structure // Laser Acceleration of Particles: AIP. N91. New York. 1982, p.154.

5. T. Weiland. Wake Field Work at DEZY // IEEE Trans. Nuel. Sci. 1985. V.NS-32. N5. P.3471-3475.

6. A. M.Sessler, S. S. Yu. Relativistic Klystron Two-Beam Accelerator// Phys. Rev. Lett. 1987. V.58. N23. P.2439-2442.

7. J. A. Nation, New Aeeelerator Coneepts // Proeeedings of the 7th International Conferenee on High-Power Particle Beams. 'Beams 88'. 1988. V.1. P.175-183.

8. P. Keining, Y. E. Jones. Annular Beam Driven High Gradient Accelerators // Proeeedings of the 7th International Conference on High-Power Particle Beams. 'Beams 88'. 1988. V.2. P.864-869.

9. V. A. Balakirev, A. O. Ostrovsky. Acoeleration of Particles with the Plasma Wave Excited by a Modulated Electron Stream // Ukr. Fiz. Zh. 1989. V.34. N.1. P.76-79 (in Russ.).

10. V. A. Balakirev, A. O. Ostrovsky. Particle Acceleration by a Modulated Electron Beam in Corrugated Cavity // Zh. Tekh. Fiz. 1989. V.59. N6. P.142-145 (in Russ.).

11. U. A. Allen, J. K. Boyd, K. R. Eppley e.a. High Gradient Accelerator Powered by a Relativistic Klystron // Collection Voprosy Atomnoi Nauki i Tekhniki (Questions of Atomio Science and Technology), ser.: Yaderno-Fizicheskiye Issledovaniya. 1990. V.7(15). P.84-90. 
12. A. O. Ostrovsky, Two-Beam Acceleration of Charged Particles in Vaccuum and Plasma Waveguide Systems // XVth International Conferenoe on High Energy Accelerators. Hamburg. 1992. Abstracts. P.95.

13. R. A. Krall, A. W. Trivelpiece. Principles of Plasma Physics. Moscow. Mir Publ. 1975. 528 p. (in Russ.)

14. L. A. Vainshtein, V. A. Solntsev. Lectures on High Frequency Electronics. Sov. Radio Publ. 1973. 400 p. (in Russ.)

15. N. G. Matsiborko, I. N. Onishenko, V. D. Shapiro, V. I. Shevchenko. On Non-Line Theory of Instability of Monoenergetic electron beam in plasma // Plasma Pysics. 1972. V.14. N6. P. 591-600. 
Table

\begin{tabular}{|c|c|c|c|c|c|c|}
\hline System parameters & \multicolumn{2}{|c|}{1} & \multicolumn{2}{|c|}{2} & \multicolumn{2}{|c|}{3} \\
\hline Drivina FEE current $\left(I_{b^{\prime}} I_{A}{ }^{\prime}\right.$ & \multicolumn{2}{|c|}{0.5} & \multicolumn{2}{|c|}{0.67} & \multicolumn{2}{|c|}{$\vdots$} \\
\hline Detuning quantity $\Delta$ & \multicolumn{2}{|c|}{0.025} & \multicolumn{2}{|c|}{0.03} & \multicolumn{2}{|c|}{0.03} \\
\hline $\begin{array}{l}\text { Maximum amplitude of } \\
\text { the electric field (Mv/m) }\end{array}$ & \multicolumn{2}{|c|}{282} & \multicolumn{2}{|c|}{385} & \multicolumn{2}{|c|}{500} \\
\hline $\begin{array}{l}\text { Initial energy of the } \\
\text { driven FiEE ( } \gamma_{0}^{\text {acc }} \text { ) }\end{array}$ & 3.4 & 5 & 3.24 & 5 & 3.24 & 5 \\
\hline Waveguide length $(\mathrm{cm})$ & 10.4 & 11.4 & 8.8 & 9.8 & 7.9 & 8.7 \\
\hline Acceleration rate (Mevim) & 35 & 27 & 43.5 & 33 & 52 & 46.5 \\
\hline
\end{tabular}

Table 2

\begin{tabular}{|c|c|c|c|c|c|c|}
\hline System parameters & \multicolumn{2}{|c|}{1} & \multicolumn{2}{|c|}{2} & \multicolumn{2}{|c|}{3} \\
\hline Driving REE current $\left(I_{b} / I_{A}\right)$ & \multicolumn{2}{|c|}{1} & \multicolumn{2}{|c|}{1.33} & \multicolumn{2}{|c|}{1.5} \\
\hline Detuning quantity $\Delta$ & \multicolumn{2}{|c|}{0.02} & \multicolumn{2}{|c|}{0.03} & \multicolumn{2}{|c|}{0.03} \\
\hline $\begin{array}{l}\text { Maximum amplitude of } \\
\text { the electric field }(\mathrm{Mv} / \mathrm{m})\end{array}$ & \multicolumn{2}{|c|}{323} & \multicolumn{2}{|c|}{483} & \multicolumn{2}{|c|}{500} \\
\hline $\begin{array}{l}\text { Initial energy of the } \\
\text { driven fiEe } \gamma_{0}^{a c c}\end{array}$ & 3.59 & 5 & 3.24 & 5 & 3. 24 & 5 \\
\hline Wavequide length (cm) & 11.6 & 12.5 & 10.5 & 10.5 & 9.6 & 10.5 \\
\hline Acceleration rate (Mev/m) & 42 & 37 & 43 & 40 & 51 & 45 \\
\hline
\end{tabular}




\section{Figure captions}

Fig. 1 Radial structure of the electric field longitudinal component $\Phi(r)$ of the cylindrical plasma waveguide; $r_{0}$ is the waveguide radius.

Fig. 2 A sketch of a plasma waveguide with drivirg and driven beams.

Fig. 3 Qualitative dispersion picture of the first three radial modes of the plasma waveguide; $\omega_{p}=\left(4 \pi m_{p} e^{2} / m\right)^{1 / 2}$ is the plasma frequency; $v_{o}$ is the initial velocity of the driving REB, $c$ is the speed of light.

Fig. $4 \quad \Pi_{1,2}$ and $\Lambda_{1,2}$ versus the parameter $r_{1} / r_{o}$. Curve 1 is for $\Pi_{1} ;$ Curve 2 is for $\Pi_{2}$; Curve 3 is for $\Lambda_{1}$; Curve 4 is for $\Lambda_{2}$.

Fig. $5 \operatorname{Im} \Gamma$ versus $\Delta$ for $r_{1} / r_{o}=0.65$. Curve 1 is for $I_{b}=0.06 I_{A}$; Curve 2 is for $I_{b}=0.5 I_{A}$; Curve 3 is for $I_{b}=0.66 I_{A} ;$ Curve 4 is for $I_{b}=2 I_{A}$.

Fig. $6 \operatorname{Im} \Gamma$ versus $\Delta$ for $r_{1} / r_{o}=0.75$. Curve 1 is for $I_{b}=0.13 I_{A}$; Curve 2 is for $I_{b}=0.66 I_{A}$; Curve 3 is for $I_{b}=I_{A}$; Curve 4 is for $I_{b}=2 I_{A}$.

Fig. $7 \operatorname{Im} \Gamma$ versus $\Delta$ for $r_{1} / r_{o}=0.81$. Curve 1 is for $I_{b}=0.1 I_{A}$; Curve 2 is for $I_{b}=I_{A}$; Curve 3 is for $I_{b}=2 I_{A} ;$ Curve 4 is for $I_{b}=4 I_{A}$.

Fig. 8 Amplitude $\mid A(\xi)(a)$ and phase $\operatorname{Arg} A(\xi)(b)$ versus the longitudinal coordinate $\xi$. 1 denotes the dependences obtained with taking into account the RF space charge; 2 corresponds to the dependences obtained without the RF space charge.

Fig. 9 Phase patterns of the driving beam for various $\xi$ values. a) $\xi=150$, b) $\xi=250$, c) $\xi=400$, d) $\xi=540$, e) $\xi=600$, f) $\xi=780$.

Fig $10 \eta, \eta_{1,2}$ versus the longitudinal coordinate $\xi$. Notation 1 corresponds to $\eta$; notation 2 is for $\eta_{1}$, notation 3 is for $\eta_{3}$.

Fig. $11|\tilde{A}(\xi)|(a)$ and $\operatorname{Arg} \tilde{A}(\xi)(b)$ versus the longitudinal coordinate $\xi$.

Fig. $12 q_{\max }^{\text {acc }}$ versus the longitudinal coordinate $\xi$.

Fig. 13 Phase patterns of the driven beam $\left(q^{a c c}, \varphi^{a c c}=\operatorname{Arg} \tilde{A}-\vartheta^{a c c}\right)$ for various $\xi$ values. a) $\xi=42$, b) $\xi=84$, c) $\xi=126$, d) $\xi=168$. 
Fig. 14 Electron momenta $q^{\text {acc }}$ versus the initial phases $\vartheta_{o}^{\text {acc }}$. a) $\xi=42$, b) $\xi=84$, c) $\xi=126$, d) $\xi=168$.

Fig. 15 Momentum of the accelerated particle versus the longitudinal coordinate $\xi$. 1) $\left.\vartheta_{o}^{a c c}=0.47, \gamma_{o}^{a c c}=5 ; 2\right) \vartheta_{o}^{a c c}=4.12, \gamma_{o}^{a c c}=3.24$.

Fig. 16 Electron momenta $q^{\text {acc }}$ versus the initial phases $\vartheta_{o}^{\text {acc }}$ for various $\xi$ values. a) $\xi=38$, b) $\xi=76$, c) $\xi=114$, d) $\xi=152$.

Fig. $17 \mu(\xi=1)$ and $\bar{\gamma}^{\text {acc }}(\xi=1)$ versus the phase interval values $\Delta \vartheta_{o}^{\text {occ }}$. 1) $\mu(\xi=1)$ curve, 2) $\bar{\gamma}^{\text {acc }}$ curve. 


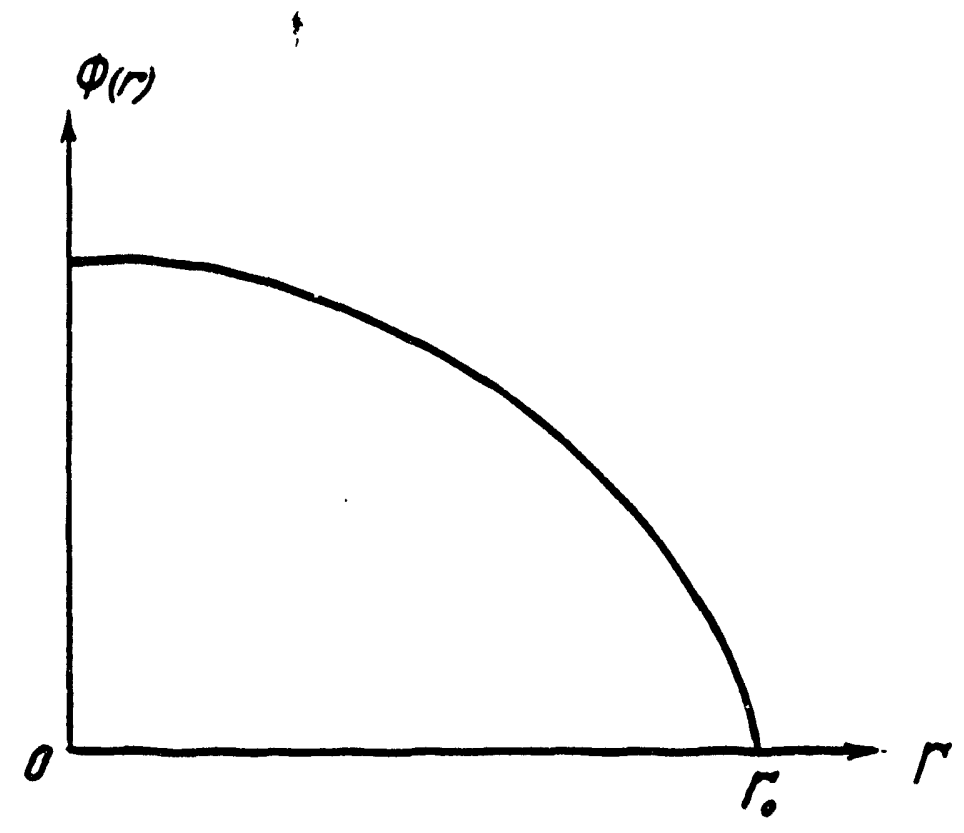

Fig. 1

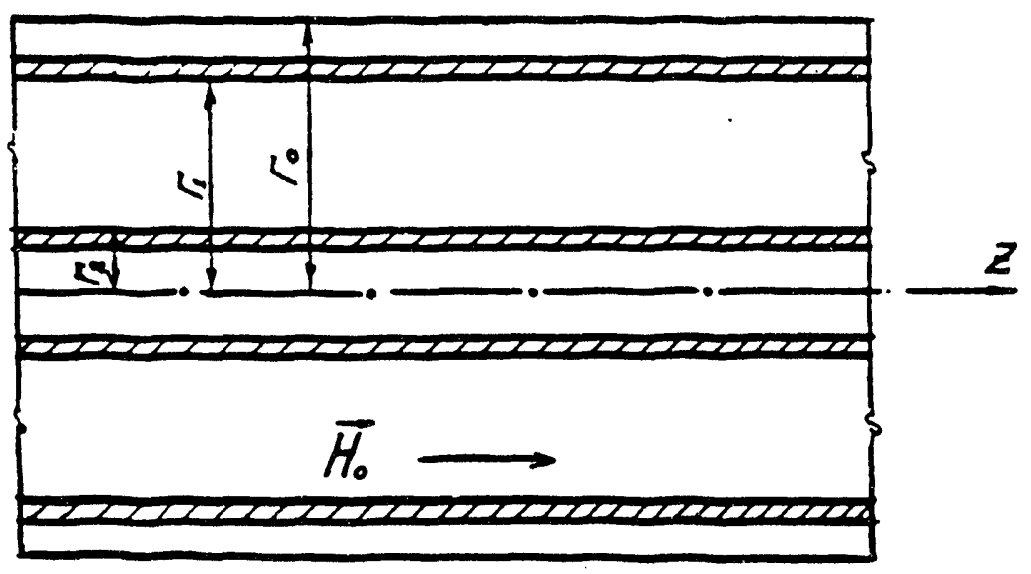

Fig. 2 


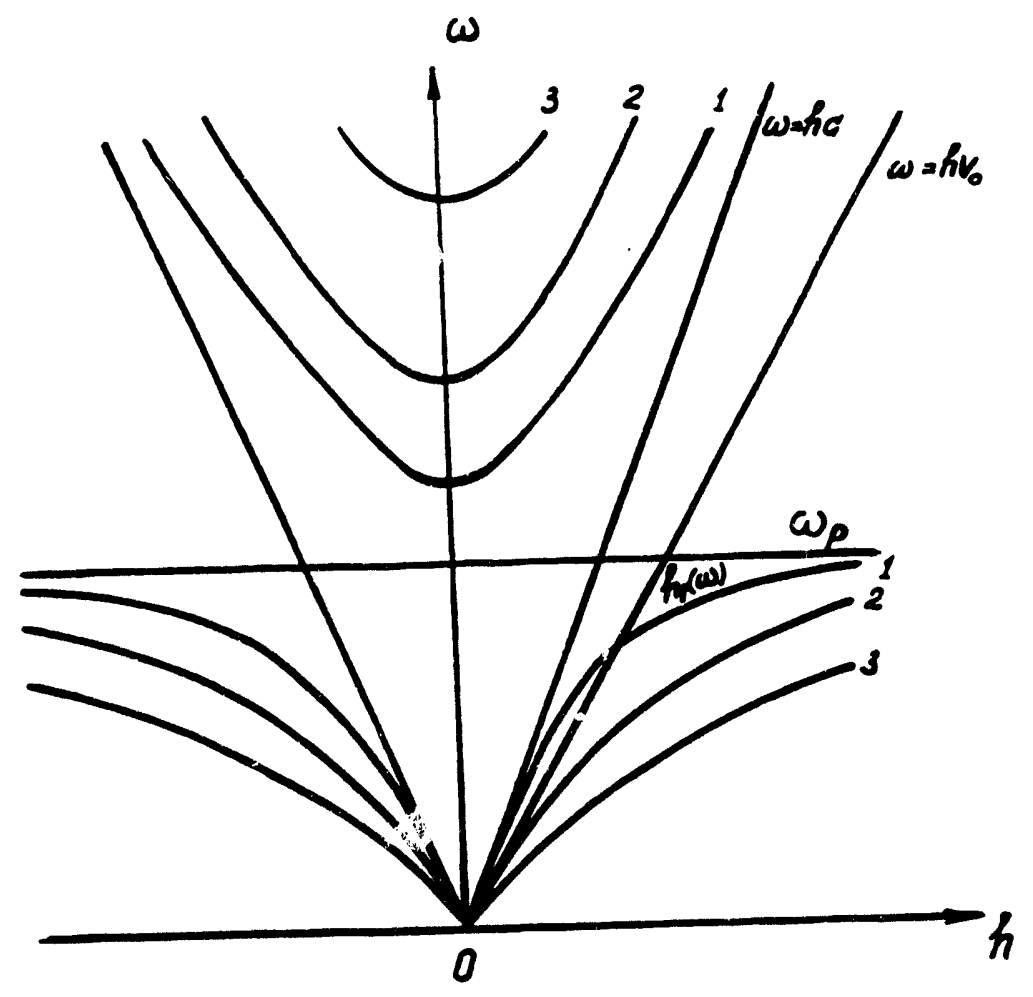

Fig. 3

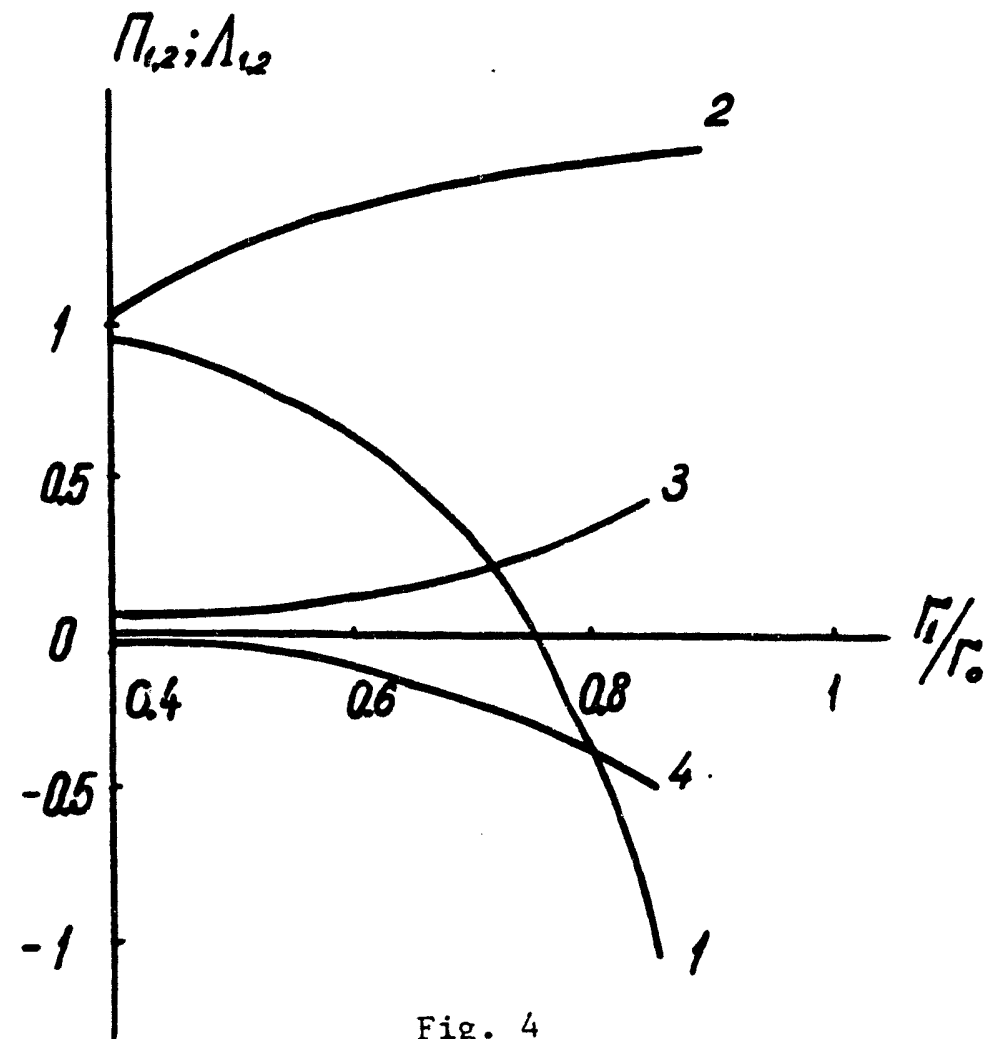




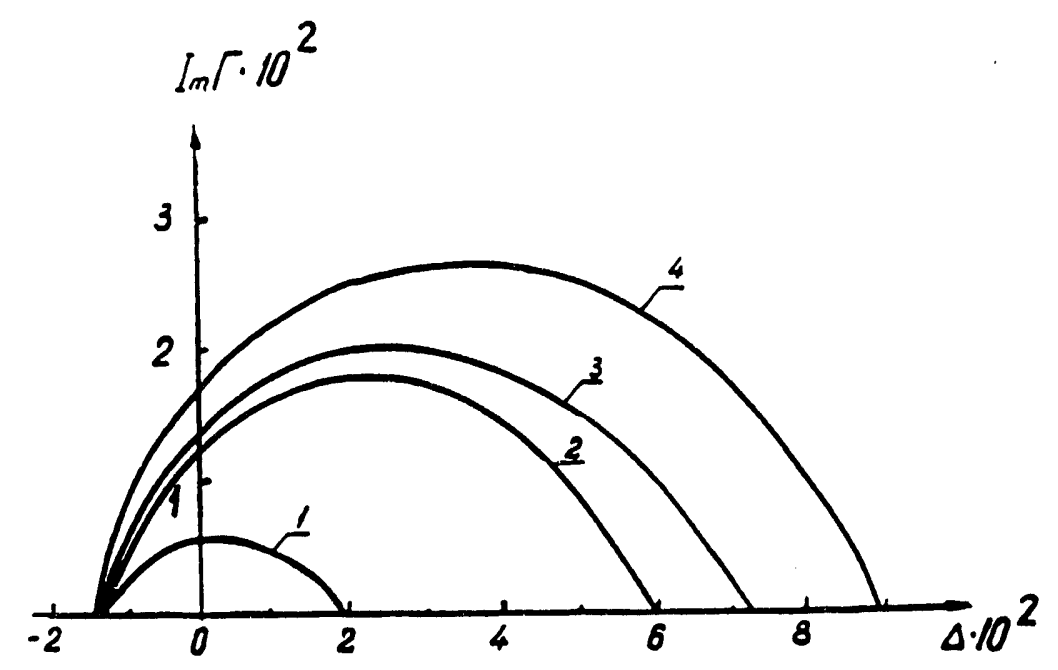

Fig. 5

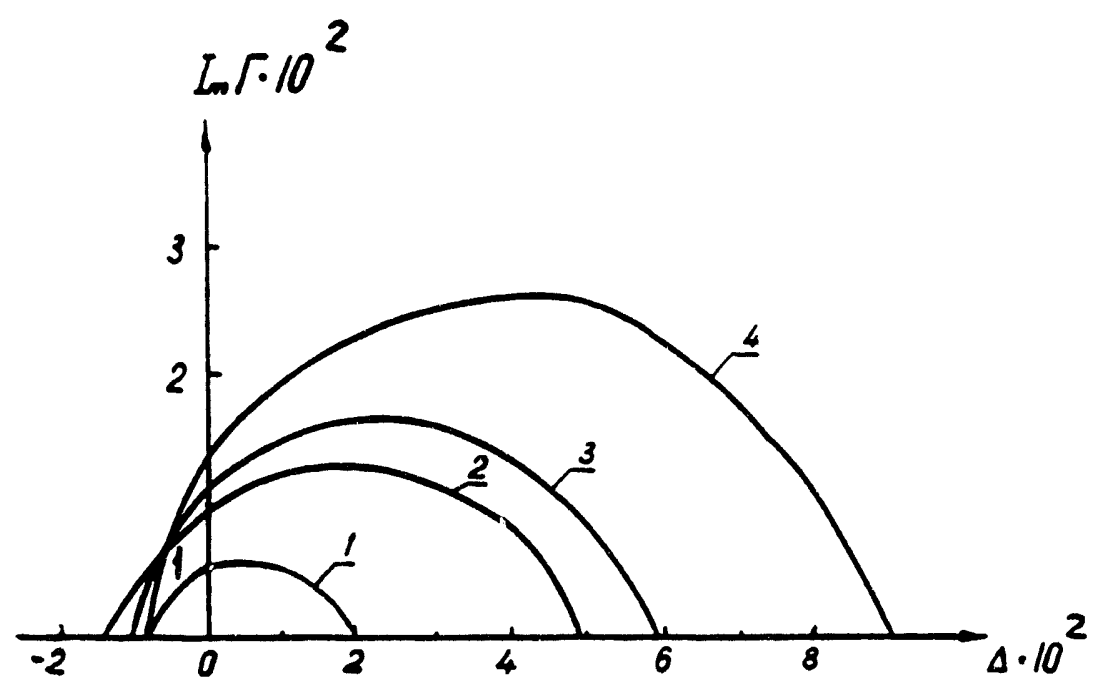

Fig. 6 


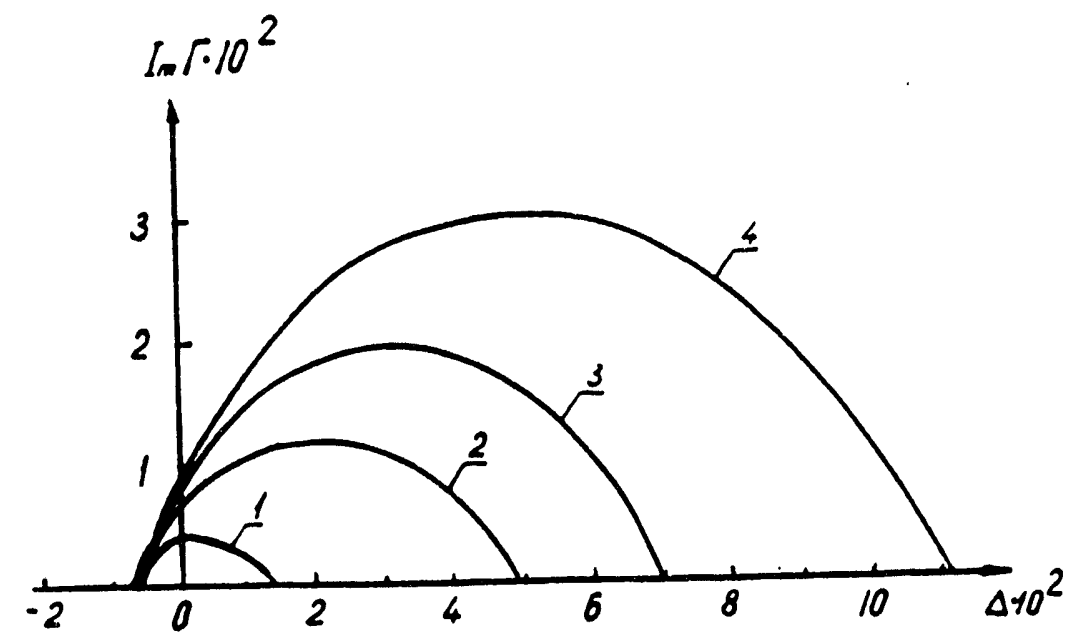

Fig. 7

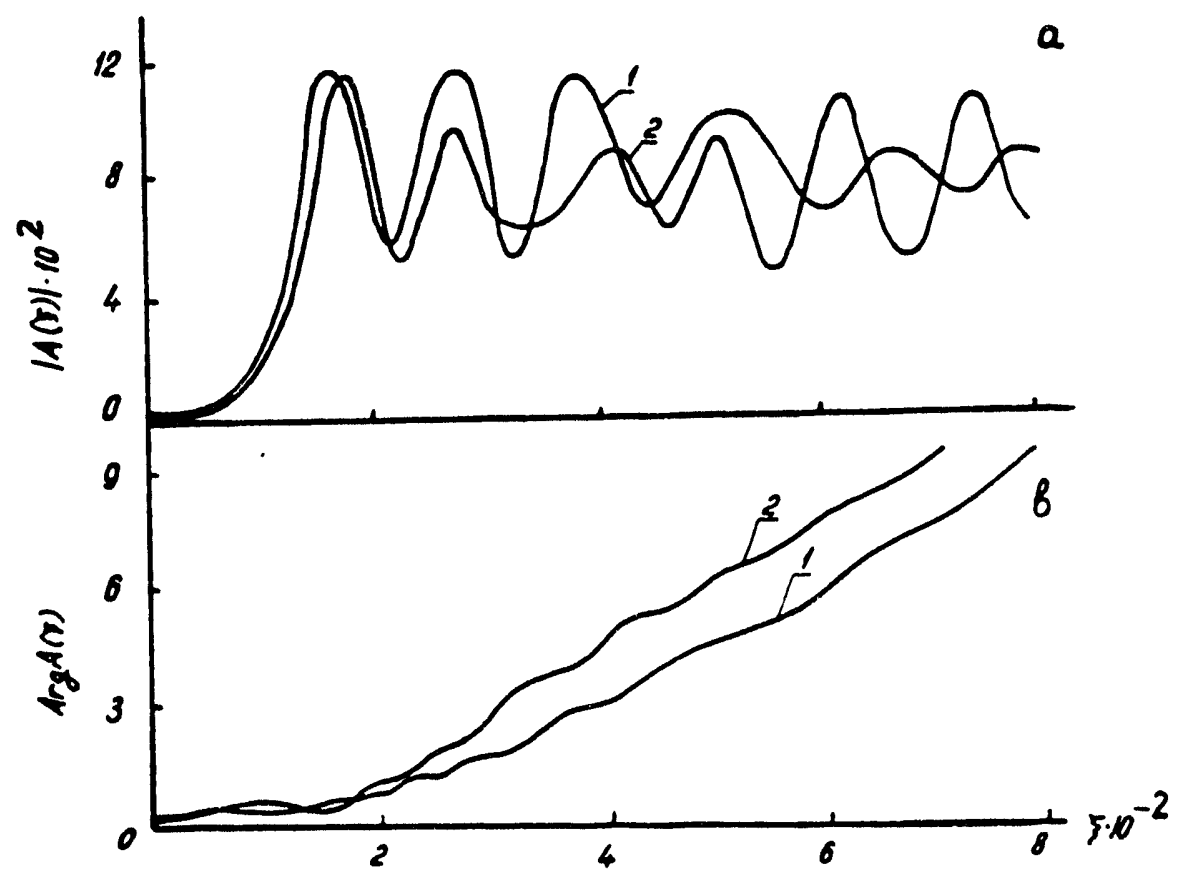

Rig. 8 

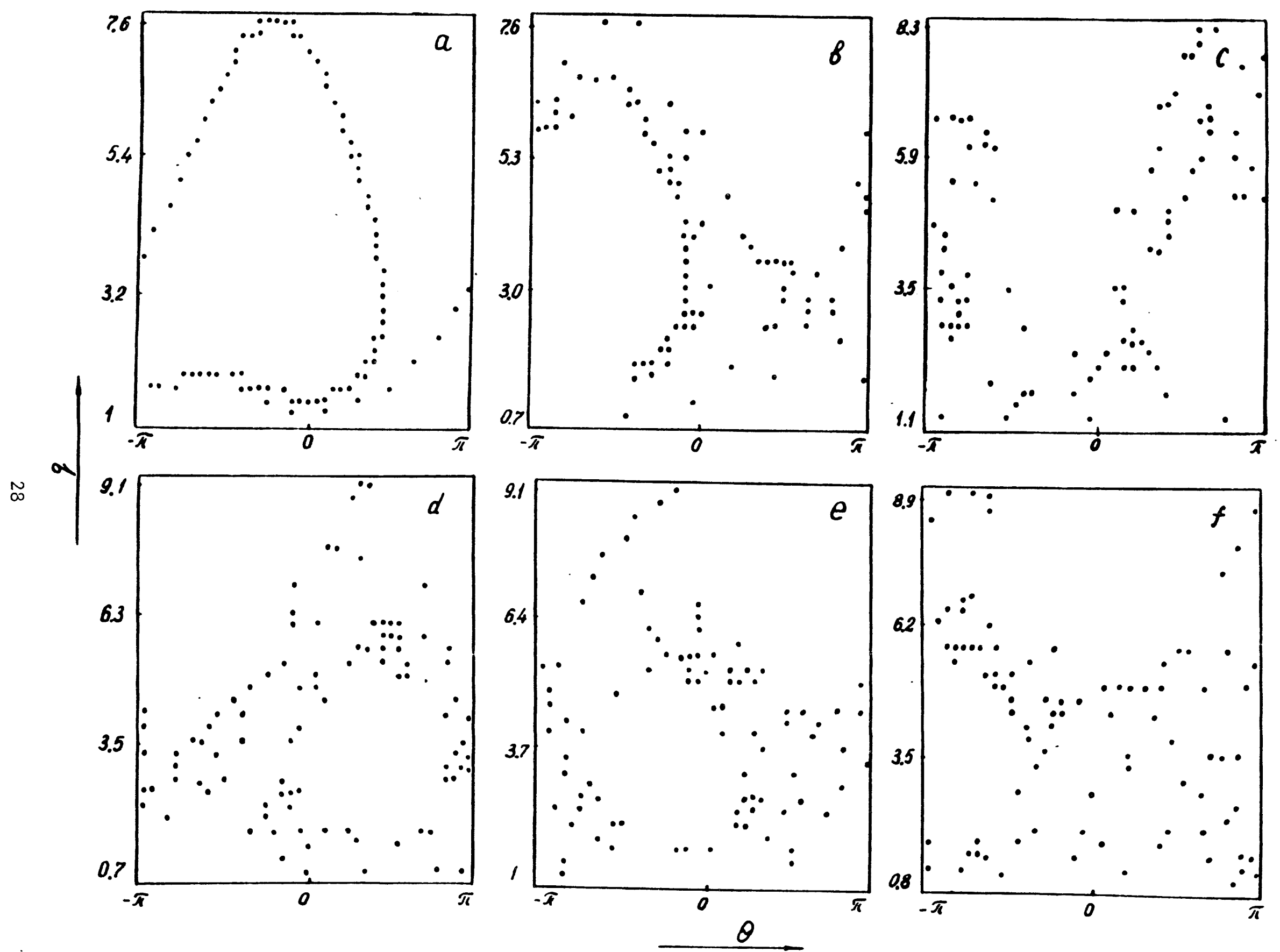

Fig.9 


\section{L.STS}

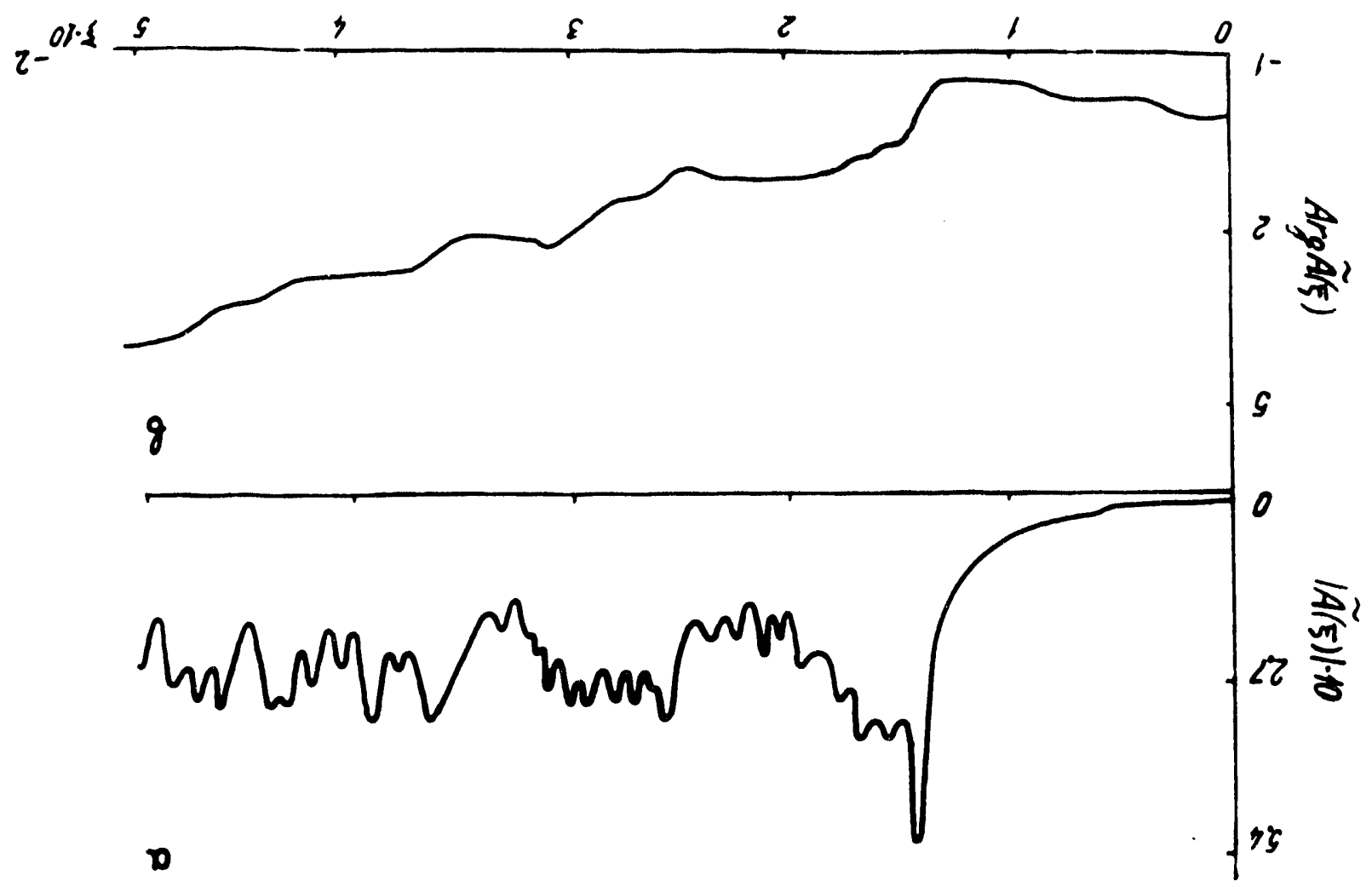

$$
\text { OL } 8 \text { 다 }
$$

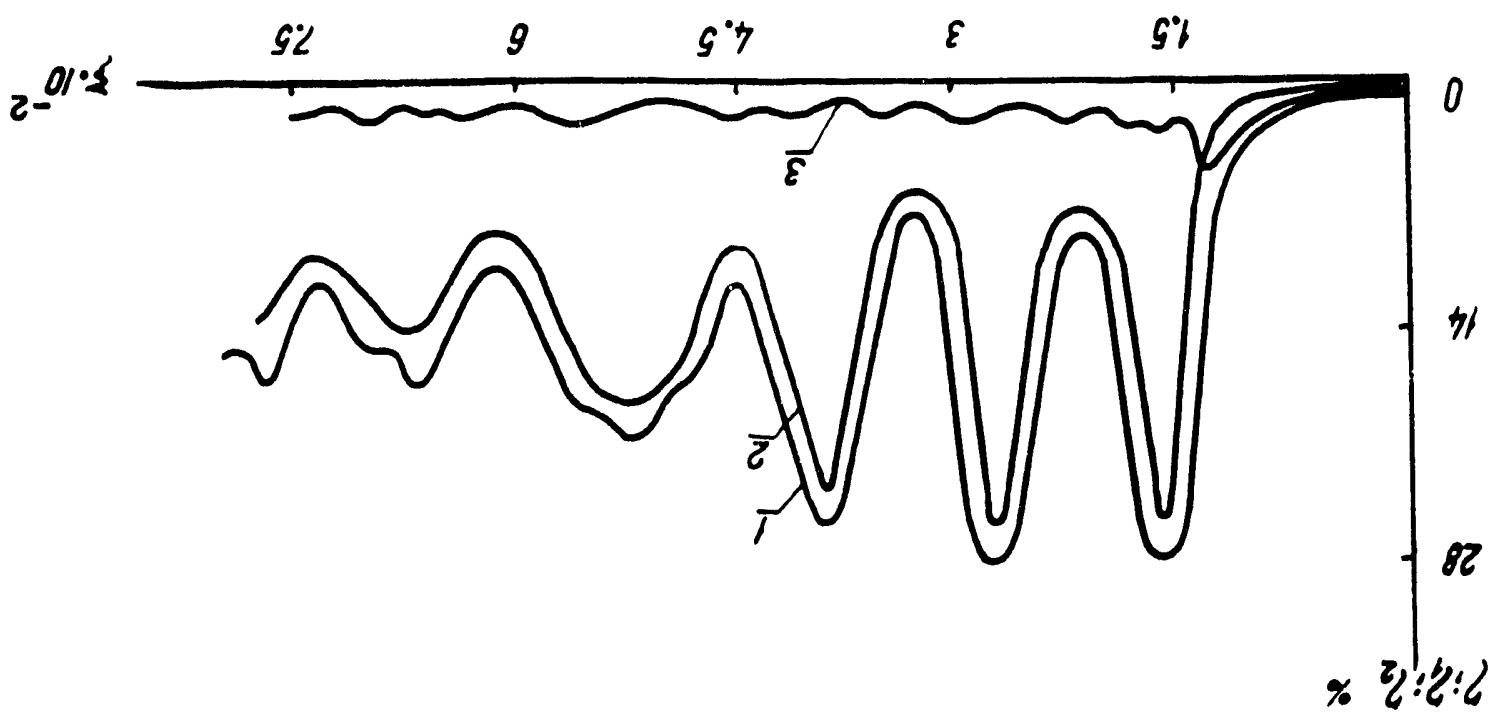




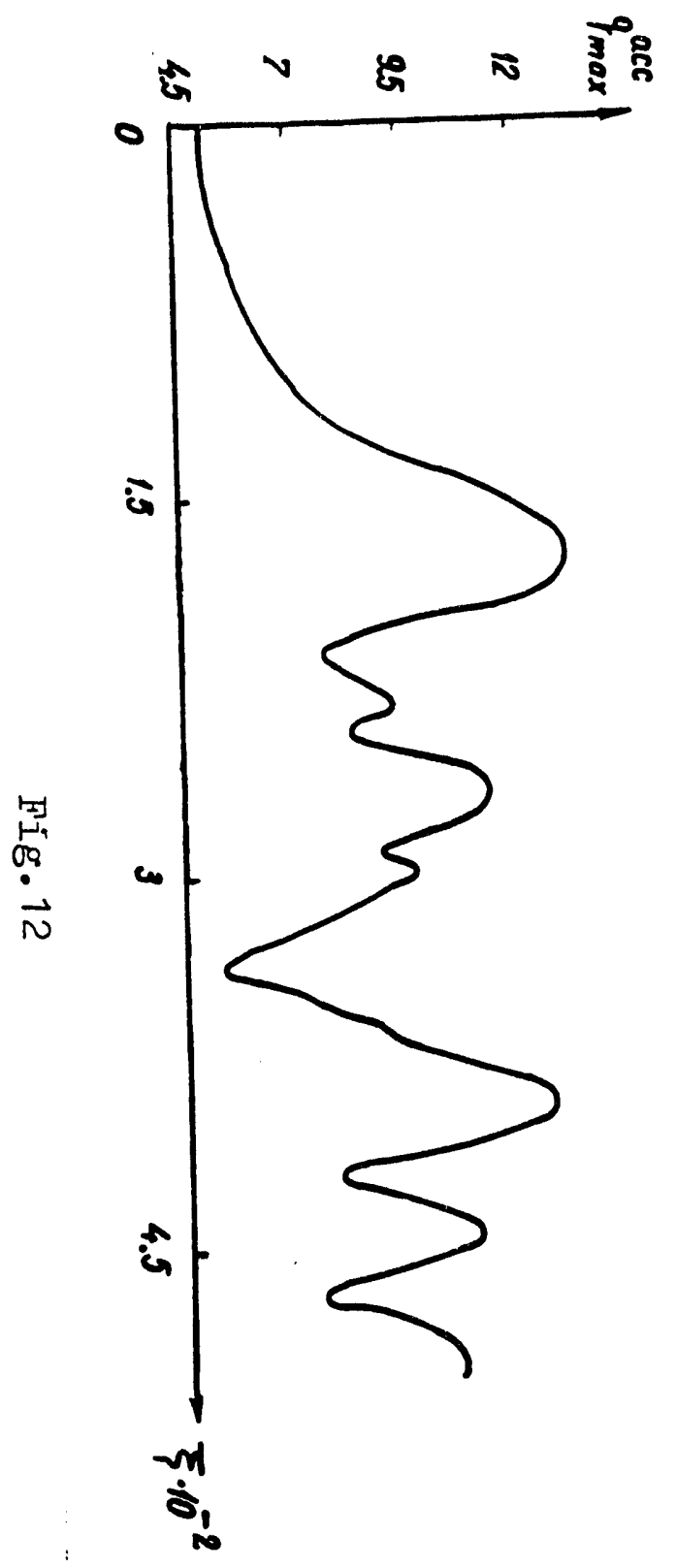



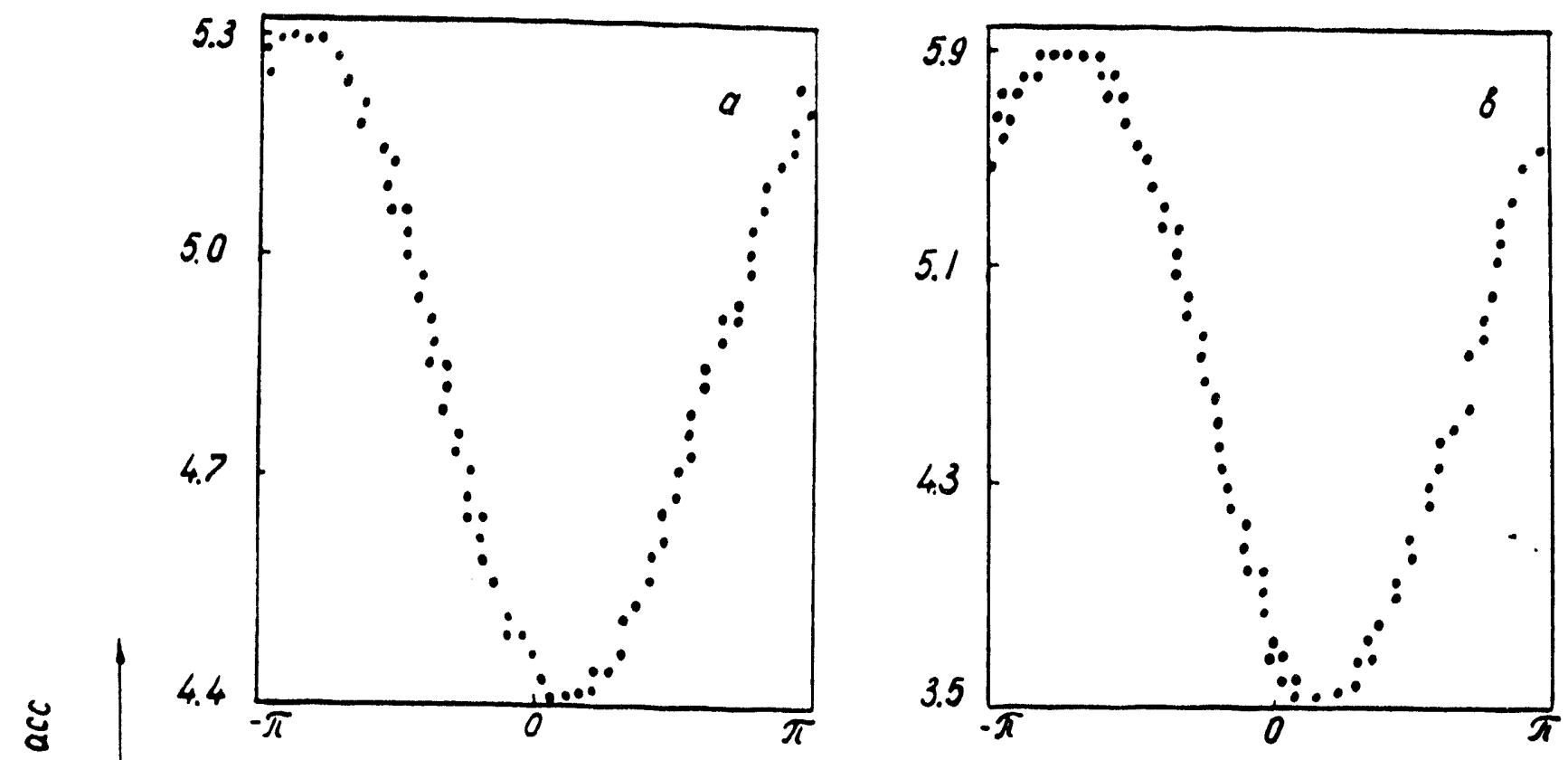

or

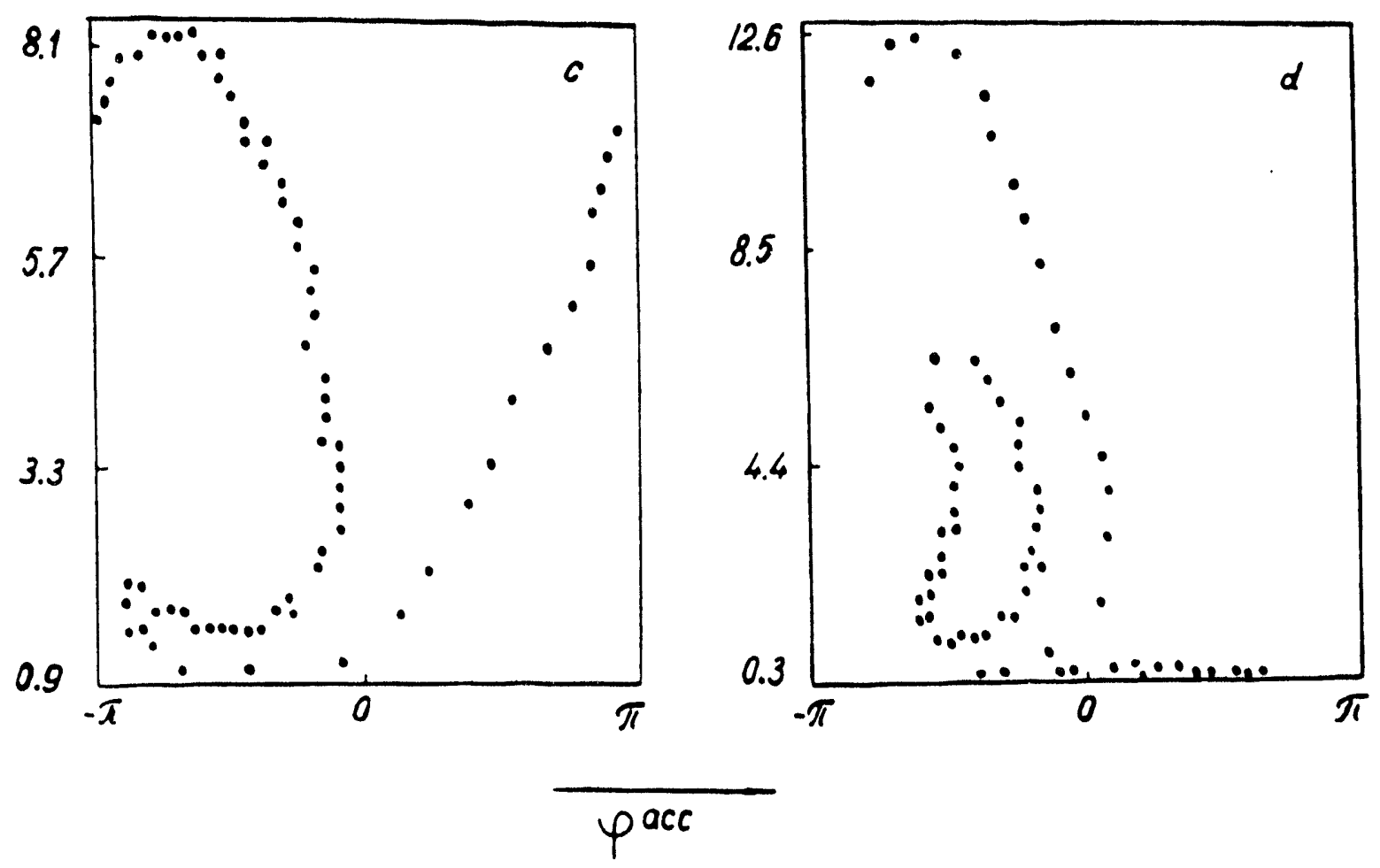

Ii. 13 


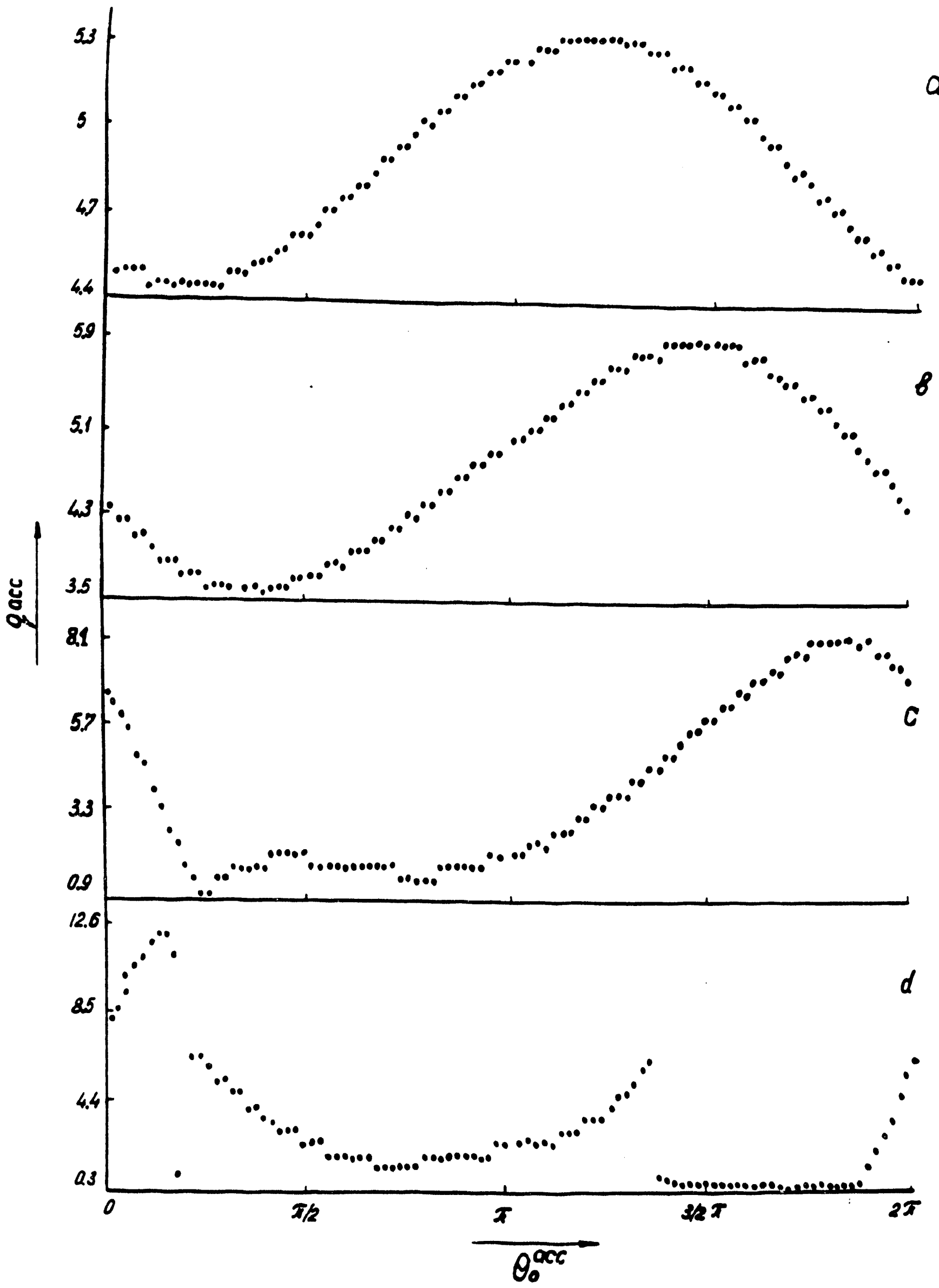

Fig. 14 


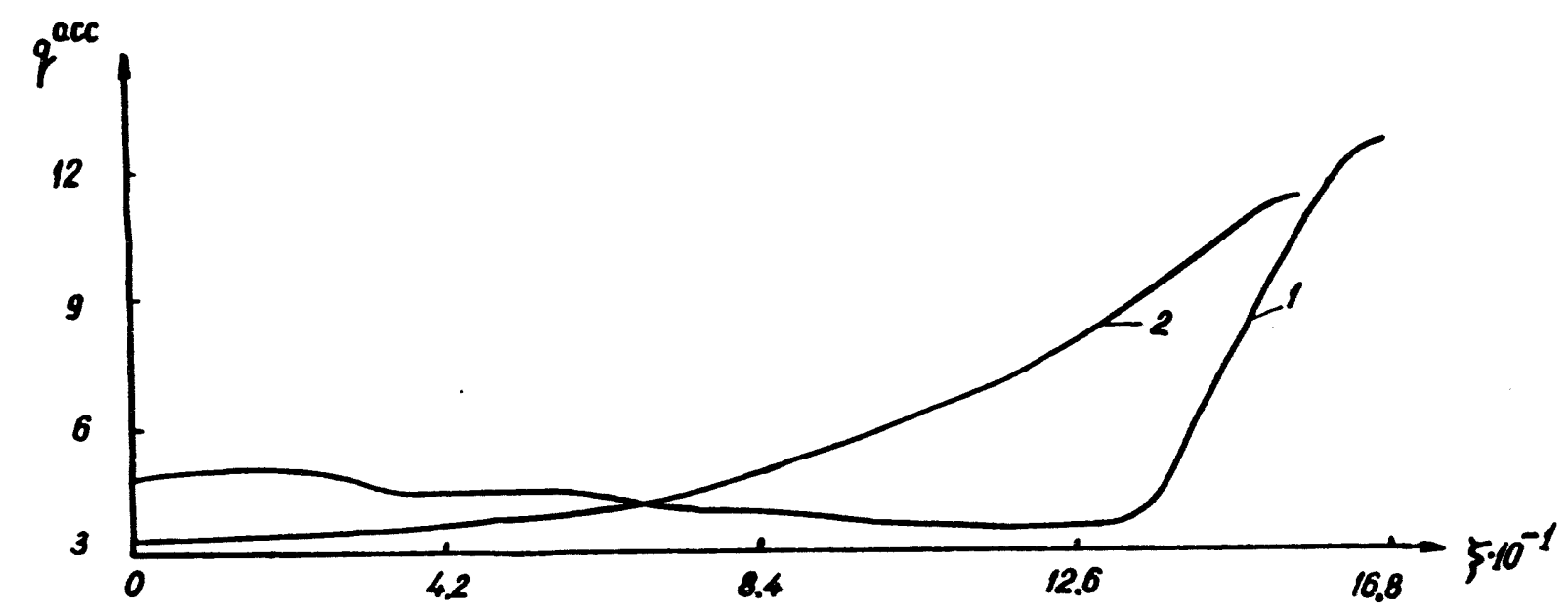

Fig. 15 


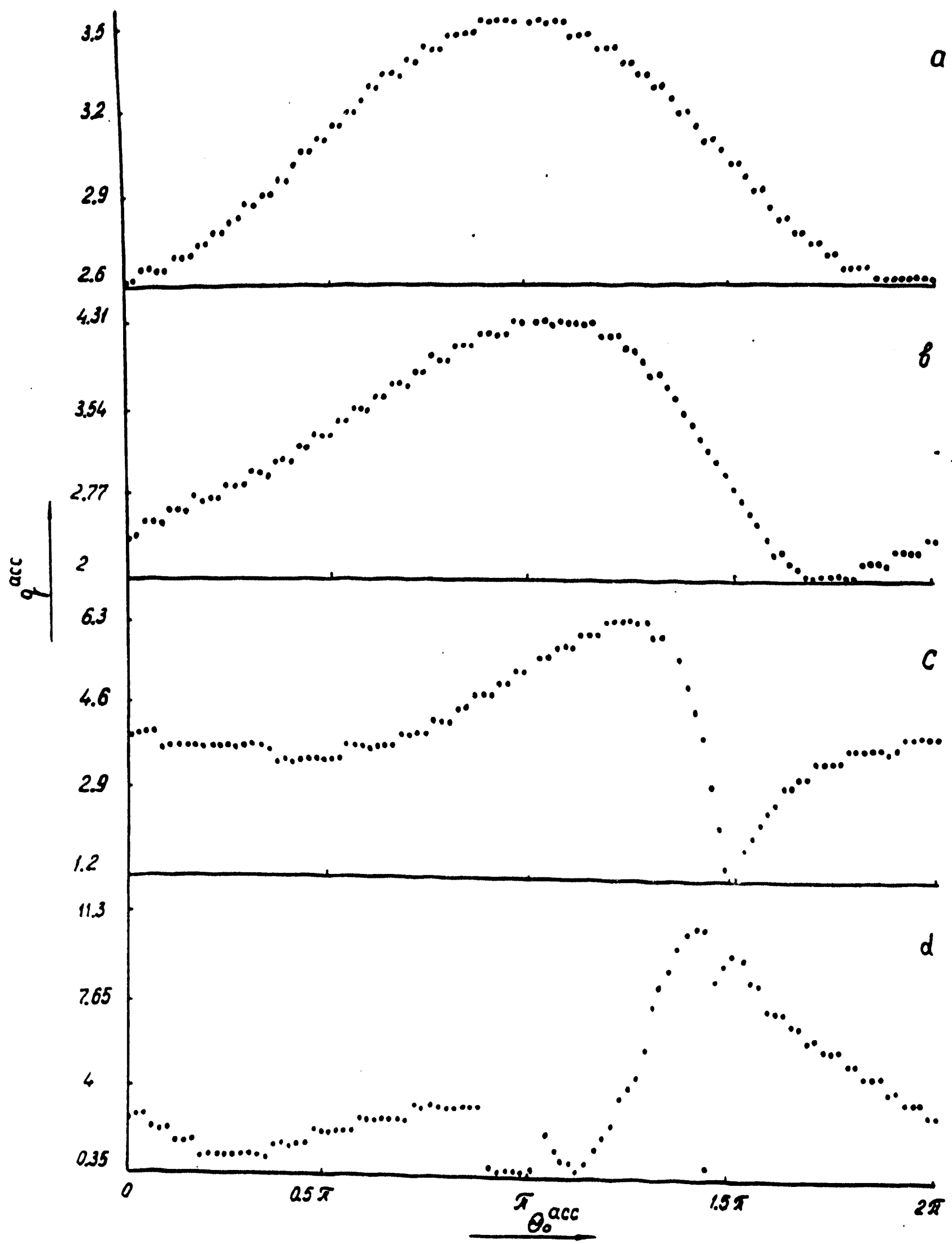

Fig. 16 


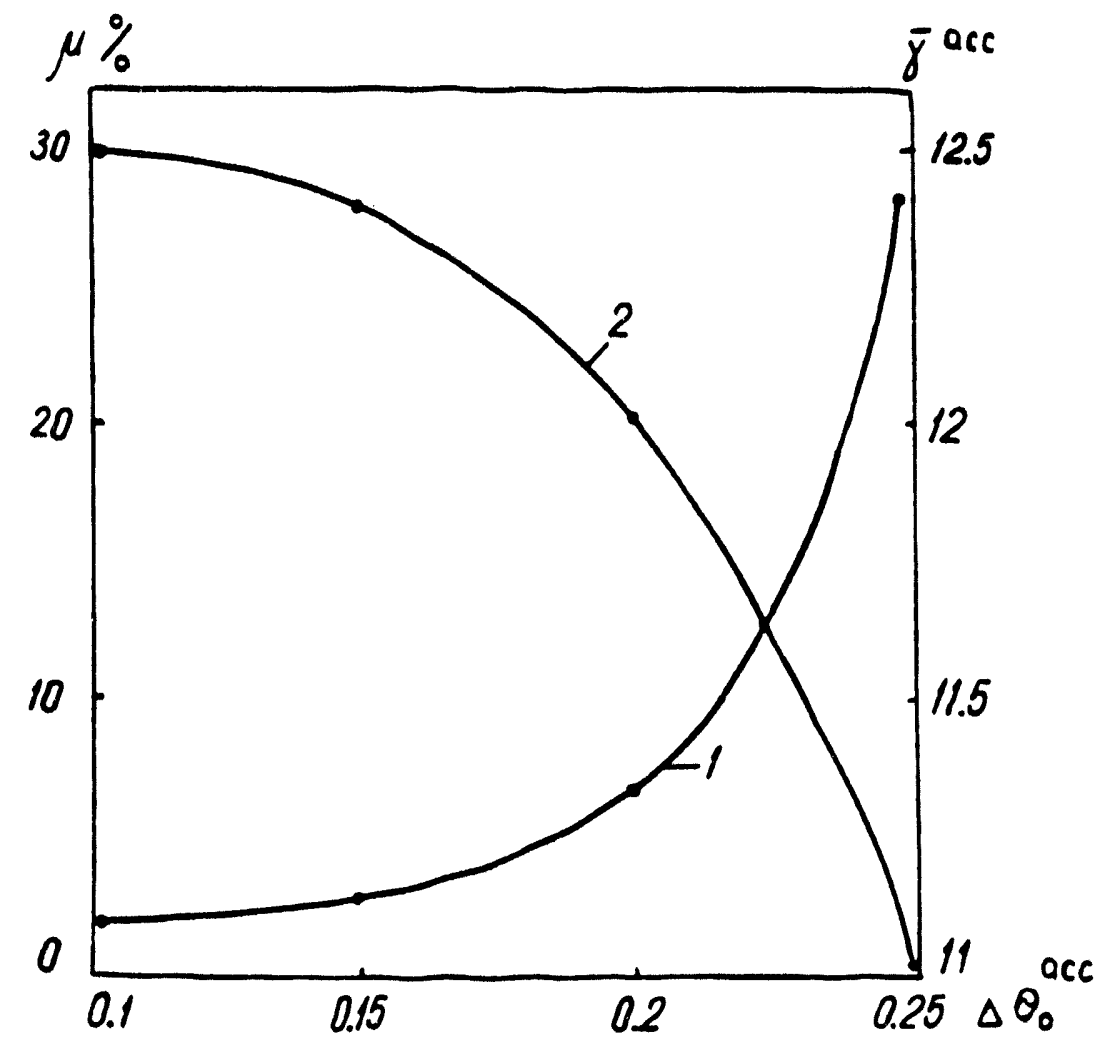

Fig. 17 

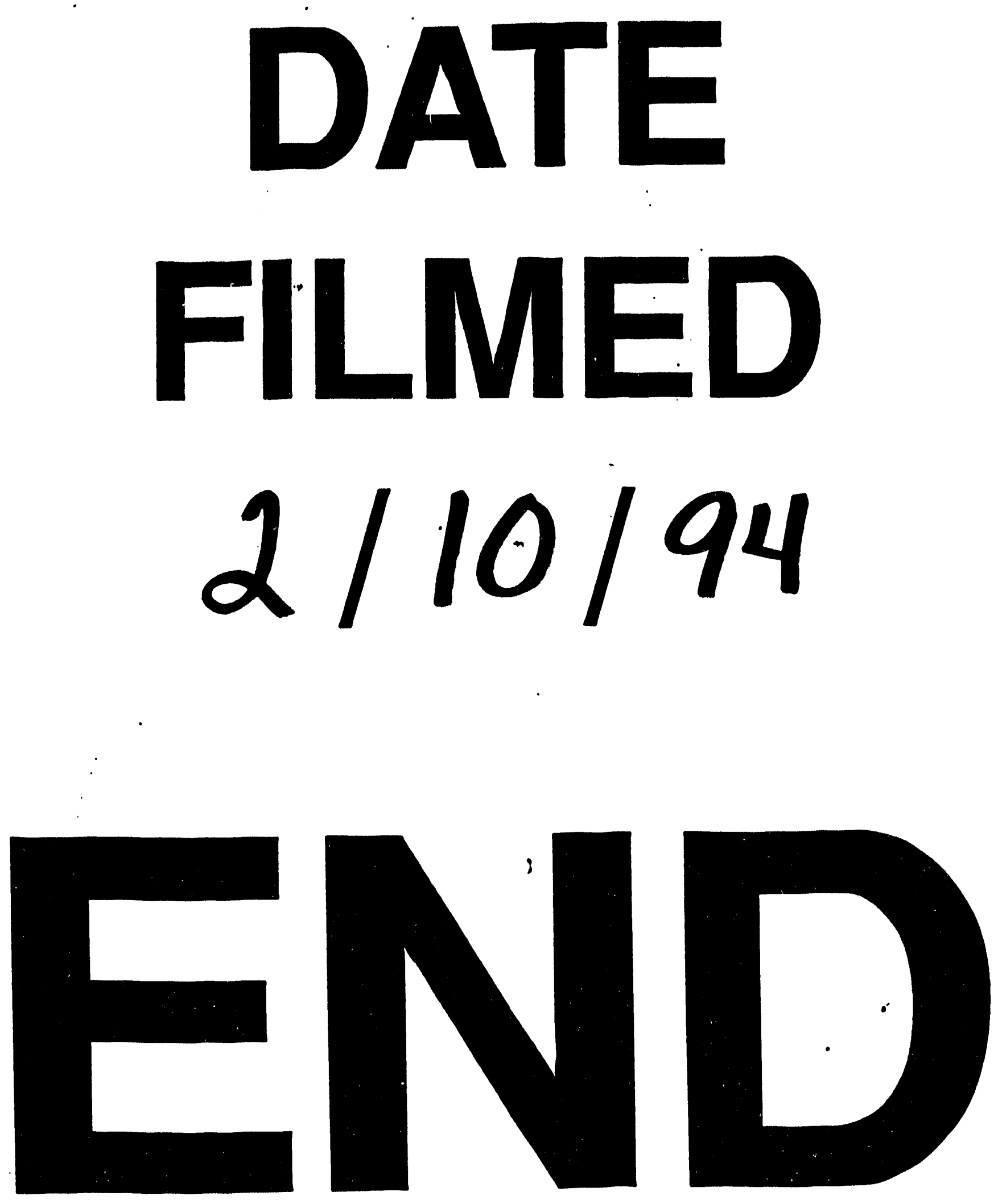
